\title{
HER2-targeted regimens after prior trastuzumab for patients with HER2-positive unresectable, locally advanced or metastatic breast cancer: a network meta-analysis of randomized controlled trials
}

\author{
Xinghui $\mathrm{Li}^{1,2 \#}$, Songwen $\mathrm{Wu}^{3 \#}$, Lijie $\mathrm{Zhang}^{1,2 \#}, \mathrm{Ji}^{\mathrm{Zhu}}{ }^{1,2}$, Binghe $\mathrm{Xu}^{4}$ \\ ${ }^{1}$ Department of Oncology, Shanghai Medical College, Fudan University, Shanghai, China; ${ }^{2}$ Department of Radiation Oncology, Fudan University \\ Shanghai Cancer Center, Shanghai, China; ${ }^{3}$ Department of Public Health, Xi'an Medical University, Xi'an, China; ${ }^{4}$ Department of Medical \\ Oncology, National Cancer Centre/National Clinical Research Centre for Cancer/Cancer Hospital, Chinese Academy of Medical Sciences and \\ Peking Union Medical College, Beijing, China \\ Contributions: (I) Conception and design: X Li, J Zhu, B Xu; (II) Administrative support: J Zhu, B Xu; (III) Provision of study materials or patients: \\ J Zhu, B Xu; (IV) Collection and assembly of data: X Li, L Zhang; (V) Data analysis and interpretation: S Wu, X Li, L Zhang; (VI) Manuscript \\ writing: All authors; (VII) Final approval of manuscript: All authors. \\ "These authors contributed equally to this work. \\ Correspondence to: Ji Zhu. Fudan University Shanghai Cancer Center, 270, Dongan Rd, Xuhui District, Shanghai 200032, China. \\ Email: leoon.zhu@gmail.com; Binghe Xu. National Cancer Centre/National Clinical Research Centre for Cancer/Cancer Hospital, Chinese \\ Academy of Medical Sciences and Peking Union Medical College, No. 17, Panjiayuan Nanli, Chaoyang District, Beijing 100021, China. \\ Email: bhxu@hotmail.com.
}

\begin{abstract}
Background: Several human epidermal growth factor receptor 2 (HER2)-targeted regimens (anti-HER2 target agent combined chemotherapy) have been introduced for the treatment of HER2-positive locally advanced or metastatic breast cancer progressed after trastuzumab. We therefore conducted a network metaanalysis to compare and rank HER2-targeted regimens in this population after trastuzumab therapy.

Methods: The electronic databases of PubMed, EmBase, Cochrane Central Register of Controlled Trials, and the websites of http://clinicaltrials.gov/ (US NIH) were systematically searched for published and unpublished randomized controlled trials (RCTs) from their inception to October, 2020. Nine treatment regimens were eligible to be included in this analysis. The primary outcomes were overall response rate (ORR), progression-free survival (PFS) and overall survival (OS), while the secondary outcomes were grade $\geq 3$ adverse events.

Results: A total of 2,104 citations were identified and 12 RCTs comprising 3,769 patients were selected for final analysis. For HER2 positive unresectable, locally advanced or metastatic patients progressed after trastuzumab therapy pyrotinib plus capecitabine ranked the highest surface under the cumulative ranking area (SUCRA) in PFS, ORR and its SUCRA in OS was higher than Trastuzumab emtansine (T-DM1). T-DM1 plus atezolizumab, pyrotinib plus capecitabine, and pertuzumab plus trastuzumab plus capecitabine had comparable SUCRA in OS (76.1\% vs. $74.5 \%$ vs. $71.2 \%)$. Six of included studies reported any grade $\geq 3$ adverse events, the prevalence of any grade $\geq 3$ adverse events in lapatinib plus capecitabine (353/683), T-DM1 (213/558), trastuzumab plus capecitabine (130/218), pertuzumab plus trastuzumab plus capecitabine (118/228), pyrotinib plus capecitabine (220/384), T-DM1 plus atezolizumab (43/132) and capecitabine (24/94) were $51.7 \%, 38.2 \%, 59.6 \%, 51.8 \%, 57.3 \%, 32.6 \%$ and $25.5 \%$, respectively. Specific adverse event characteristics related to different HER2-targeted regimens need to be well known ahead and managed during the therapy.
\end{abstract}

Conclusions: The results indicated that for HER2 positive breast cancer with previous trastuzumab

\footnotetext{
^ ORCID: 0000-0001-7134-9419.
} 
therapy pyrotinib plus capecitabine was probably more efficacious in PFS and ORR. T-DM1 plus atezolizumab, pyrotinib plus capecitabine and pertuzumab plus trastuzumab plus capecitabine have comparable effect on OS improvement and all of them were likely better than T-DM1. The risk of grade $\geq 3$ adverse events for specific treatment regimens were also provided.

Keywords: Breast neoplasms; molecular targeted therapy; lapatinib; pyrotinib; ado-trastuzumab emtansine

Submitted Jun 09, 2020. Accepted for publication Dec 15, 2020.

doi: $10.21037 / \mathrm{atm}-20-5149$

View this article at: http://dx.doi.org/10.21037/atm-20-5149

\section{Introduction}

Breast cancer (BC) is the most common cancer diagnosed in females globally which is a heterogeneous disease across varies subtypes including luminal, human epidermal growth factor receptor 2 (HER2) positive, and basal-like tumors (1-4). The prognosis and treatment strategies for BC depend on the stage, grade, proliferation, the expression of HER2 and the hormonal receptors and other exploring biomarkers. The over-expression of HER2 account for nearly $15-20 \%$ of $\mathrm{BC}$ which associated with poor prognosis $(5,6)$. The anti-HER2 monoclonal antibody, trastuzumab, combined regimen has been approved for metastatic BC since 1998 (7). Trastuzumab combined with chemotherapy have been proved to provide additional benefit for metastatic HER2 positive BC in multiple studies (8-10). In another institutional-based review one-year survival rate in HER2 positive $\mathrm{BC}$ with or not with trastuzumab in first line were $80.6 \%$ and $70.2 \%$ separately which also showed that trastuzumab had increased the survival rate and improved the prognosis of HER2 positive metastatic BC (11). However, resistance to trastuzumab could be detected by HER reprogramming after long-term trastuzumab exposure in cell lines $(12,13)$. An alternative or combined inhibition of ErbB family signal pathway might provide additional benefit for patients who progress after trastuzumab therapy (14-16).

Hermine and GBG 26 had showed trastuzumab continuation beyond progression with changing chemo agent could bring additional benefit for patients $(17,18)$ however the result of CLEOPATRA and an Italy real world study consistently indicated that patients with previous trastuzumab seemed to gain less benefit from further trastuzumab combined regimen $(19,20)$. At the same time new target agents including tyrosine kinase inhibitors (TKIs) and Antibody-drug conjugate (ADC) had been introduced into the HER2 positive breast cancer field. Geyer et al.
(EGF100151 study) showed that lapatinib plus capecitabine had super benefit than lapatinib alone in PFS which were 8.4 versus 4.4 months $(\mathrm{P}<0.001)(21)$ and then lapatinib plus capecitabine was regarded as one of the second line treatment choices and experiment control for the next following several new HER2-targeted agents explored in HER2 positive BC. EMLIA study compared Trastuzumab emtansine (T-DM1) and lapatinib plus capecitabine and the PFS was 9.6 versus 6.4 months $(\mathrm{P}<0.001)(22)$. Pyrotinib is a newly-developed irreversible pan-ErbB receptor tyrosine kinase inhibitor. In a phase 2 study pyrotinib plus capecitabine showed overall response rate (ORR) of $78.5 \%$ and progression-free survival (PFS) of 18.1 months compared to lapatinib plus capecitabine (ORR 57.1\% and PFS 7.0 months) (23). Based on this phase 2 study result pyrotinib initiated two phase 3 trials comparing pyrotinib plus capecitabine to capecitabine alone or lapatinib plus capecitabine respectively. Both trials showed superior ORR and PFS in pyrotinib plus capecitabine arm than in control arm $(24,25)$. With multiple available data and evidence, sometimes it is a true difficulty for clinicians to make a treatment decision in the real clinical practice when facing HER2 positive BC with previous trastuzumab therapy. The limitation of the studies mentioned above is that they usually compared only two specific interventions and the result can only give information on these two arms and they cannot provide information on efficacy from a whole picture perspective of all available options so we performed this analysis in order to provide valuable information for this clinical dilemma.

In this analysis we aimed to compare various HER2targeted regimens for patients with HER2-positive unresectable, locally advanced or metastatic BC after trastuzumab therapy by using a network meta-analysis (26) on the basis of directly and indirectly evidence.

We present the following article in accordance with the 
PRISMA reporting checklist (available at http://dx.doi. org/10.21037/atm-20-5149).

\section{Methods}

\section{Search strategy and selection criteria}

The Preferred Reporting Items for Systematic Reviews and Meta-Analysis Statement was applied to guide and report this network meta-analysis (27). Randomized controlled trial (RCT) which investigated the efficacy and safety of different HER2-targeted regimens for patients with HER2positive unresectable, locally advanced or metastatic BC after trastuzumab therapy was eligible in this study. The electronic databases of PubMed, EmBase, Cochrane Central Register of Controlled Trials were systematically searched for eligible study from their inception to October, 2020, and no restrictions were placed on published language and status, and the core search terms including "breast cancer", "advanced", "HER-2", "second-line", and combined with a list of HER2-targeted regimens. The details of search strategy in each database are displayed in Appendix 1. Furthermore, the website http://clinicaltrials.gov/ (US $\mathrm{NIH}$ ) was reviewed for ongoing trials. If any trial met the inclusion criteria and related results had been published as we know it would be added manually. The reference lists of retrieved studies were also reviewed to identify any new eligible study.

Two reviewers independently conducted literature search, study selection following a standardized approach, and any disagreement between two reviewers was resolved by group discussion until a consensus was reached. Study was eligible in this study if they met the following inclusion criteria: (I) patients: HER2-positive unresectable and locally advanced or metastatic BC after trastuzumab therapy; (II) intervention and control (including but not limited to): lapatinib plus capecitabine, capecitabine plus trastuzumab, T-DM1, neratinib, lapatinib plus vinorelbine, pertuzumab plus trastuzumab plus capecitabine, pyrotinib plus capecitabine, and capecitabine; (III) outcomes: the primary endpoints were ORR, PFS and overall survival (OS), while the secondary endpoints were grade $\geq 3$ adverse events reported in the included studies; and (IV) study design: the study had to be RCT design. The details of exclusion criteria are listed as follows: (I) previous treatment strategies including T-DM1, or other HER2-targeted tyrosine kinase inhibitors (lapatinib, neratinib, pyrotinib); (II) number of prior trastuzumab therapy $\geq 2$; and (III) non-RCT design.

\section{Data collection and quality assessment}

The extracted items from eligible studies included first author's surname or study's specific name, publication year, sample size, mean age, crossover permitted, Eastern Cooperative Oncology Group (ECOG), positive for estrogen receptor, progesterone receptor, or both, time since initial diagnosis, time since first metastases, metastatic in brain, visceral disease, intervention, control, and reported outcomes. The methodological quality of each trial was assessed by using the JADAD scale, which on the basis of randomization, blinding, allocation concealment, withdrawals and dropouts, or use of intention-to-treat analysis, and the scoring system of each trial ranged from $0-5$ (28). The data collection and quality assessment were performed by two reviewers independently, and conflicts between reviewers were examined and adjudicated by an additional reviewer reviewing the original study.

\section{Statistical analysis}

The treatment effects of various HER2-targeted regimens used hazard ratios (HRs) for OS or PFS, and odds ratios (ORs) for ORR and grade $\geq 3$ adverse events. A network meta-analysis was conducted to compare various interventions on the basis of direct and indirect comparisons (29). The loop-specific approach was applied to assess the difference between direct and indirect estimates for a specific comparison in the loop (30). The design-by treatment interaction inconsistency model was used to check the assumption of consistency in the entire network (29). Moreover, the inconsistent model was applied to analyze data owing to the heterogeneity across included studies. Then the surface under the cumulative ranking area (SUCRA) probabilities was applied to compare and rank the treatment strategies for each investigated outcome (31). Finally, the small-study effects for each outcome were assessed by using the comparison-adjusted funnel plots (32). All of analyses in this study were conducted by using STATA software (Version 10.0; StataCorp, Texas, United States of America).

\section{Results}

\section{Literature search}

An initial electronic search yielded 2,104 citations from electronic databases, and 1,267 articles were retained after duplicate removed. Then 52 potentially eligible articles 


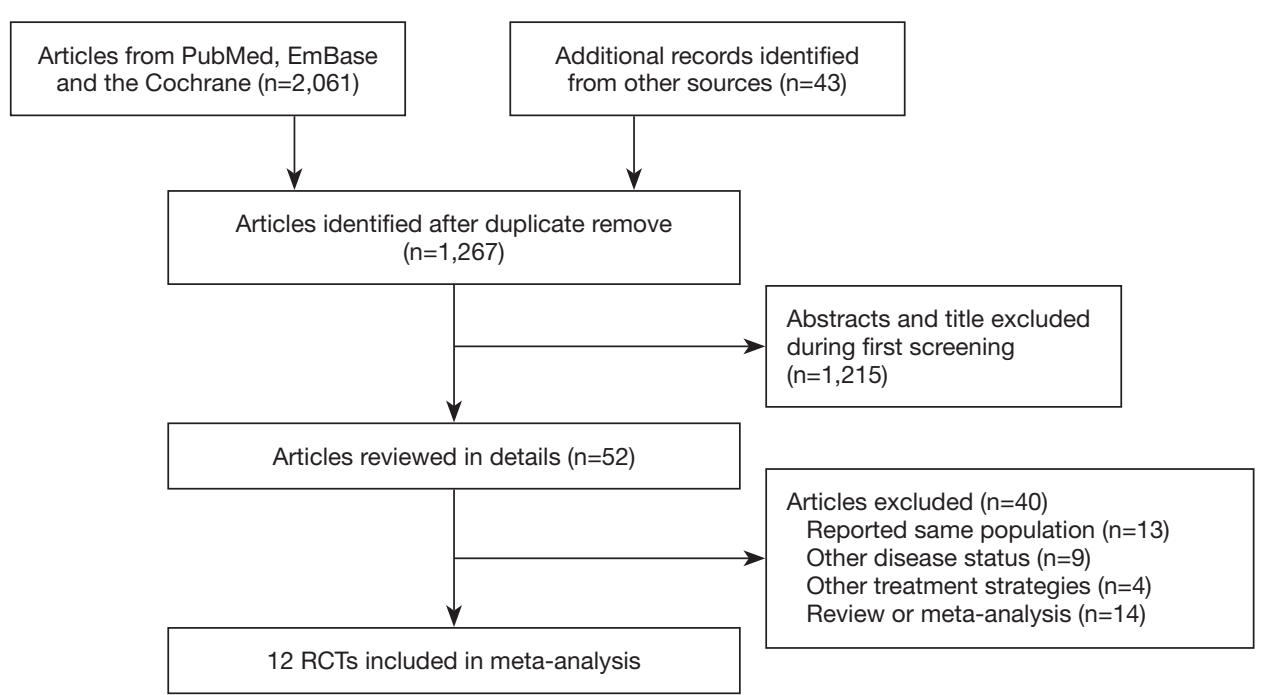

Figure 1 The details regarding the literature search and study selection process.

were retrieved in full-text evaluations after irrelevant articles excluded. After this, 40 studies were excluded owing the following reasons: reported same population $(n=13)$, patients at other disease status $(\mathrm{n}=9)$, other treatment strategies $(n=4)$, and review or meta-analysis $(n=14)$. There was no new eligible study by reviewing the reference lists of these studies. Finally, a total of 12 RCTs were selected for final analysis (17,21-25,33-38), and the details of studyselection process are presented in Figure 1.

\section{Study characteristics}

The baseline characteristics of included studies and patients are summarized in Table 1. All included trials ranged from 2006-2020, and the data from Pheobe study was based on unpublished data which had been reported as oral presentation on 2020 ASCO. The mean age of included patients ranged from 47.4-58.0 years and the population size of different trials was from 86-991 patients. The quality of included trials was assessed by using the JADAD scale, 3 trials scored 5, 7 trials scored 4 , and the remaining 2 trials scored 3 (Table 1). In the Network of comparisons, the nodes were weighted according to the contribution of specific regimen and the thickness of lines connecting different regimens represents the number of studies that were used to evaluate each pair of regimens (Appendix 2). The edges were weighted according to the precision of the direct estimate for each pair-wise comparison (Appendix 2).

\section{ORR}

The network compared various HER2-targeted regimens' effects on ORR was presented in Figure S1 of Appendix 2. An inconsistency plot was produced to assume the loopspecific heterogeneity estimate, the exp (IF). The loopspecific heterogeneity inconsistency plot is shown in Figure S2 of Appendix 2, and the result of global inconsistency was not assessed due to insufficient observations. The effects of various HER2-targeted regimens on ORR were ranked with SUCRA probabilities (\%). The SUCRA probability of pyrotinib plus capecitabine was $91.2 \%$ which ranked the highest indicating that pyrotinib plus capecitabine was most likely to increase ORR among all regimens (Figure 2). The details of pair-wise comparisons ORR were listed in https://cdn.amegroups.cn/static/public/atm-20-5149-1. xlsx. Pyrotinib plus capecitabine trends better than all other treatments however the comparison was not statistically significant when compared to pertuzumab plus trastuzumab plus capecitabine, T-DM1 and T-DM1 plus atezolizumab. The comparison-adjusted funnel plot used to assess publication bias and to determine the presence of small-study effects did not suggest any publication bias (Appendix 2).

\section{PFS}

The network compared various HER2-targeted regimens on PFS was presented in Figure S4 of Appendix 2. The loopspecific heterogeneity inconsistency plot is shown in Figure S5 of Appendix 2, and the result of global inconsistency was 


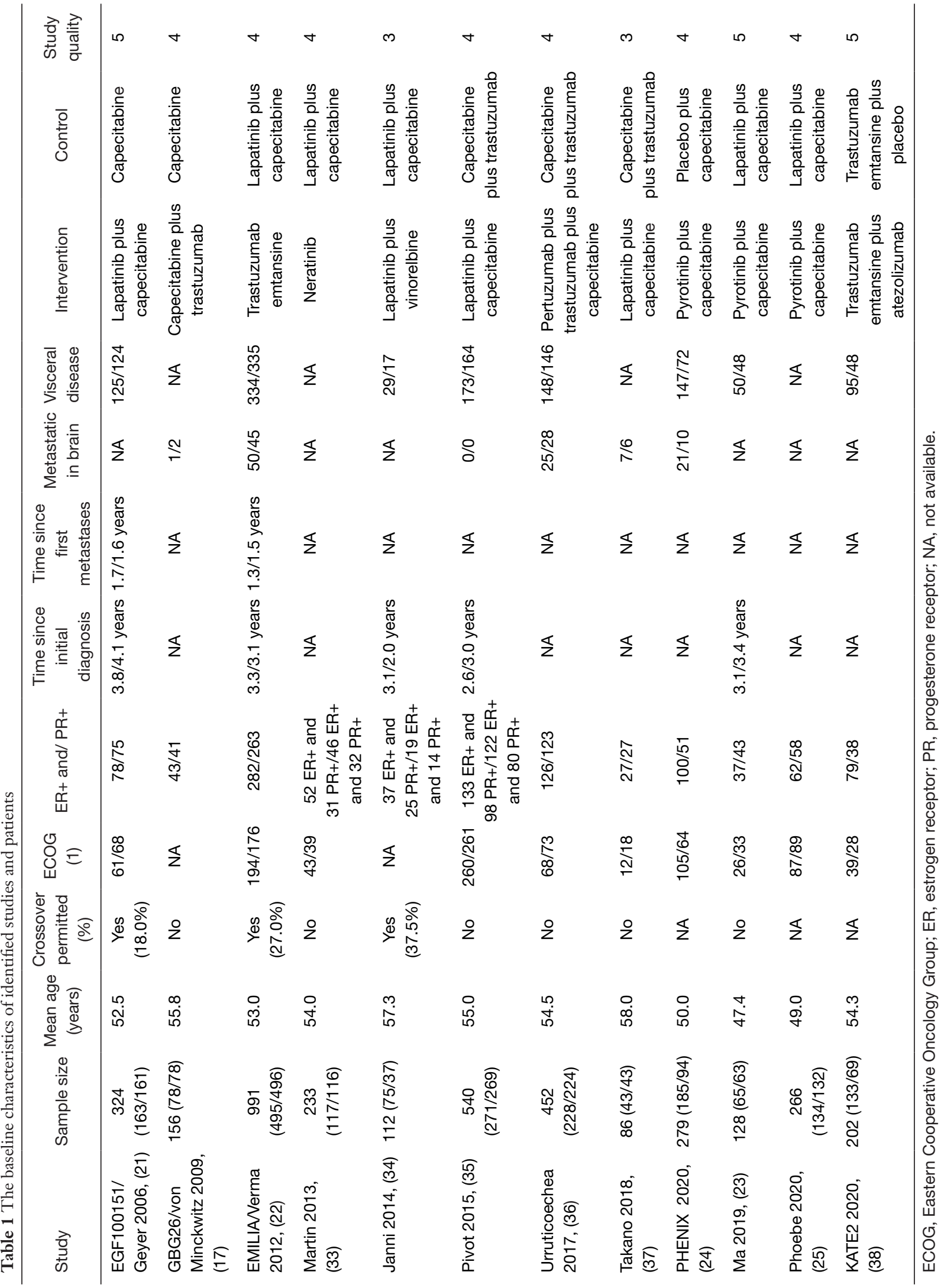



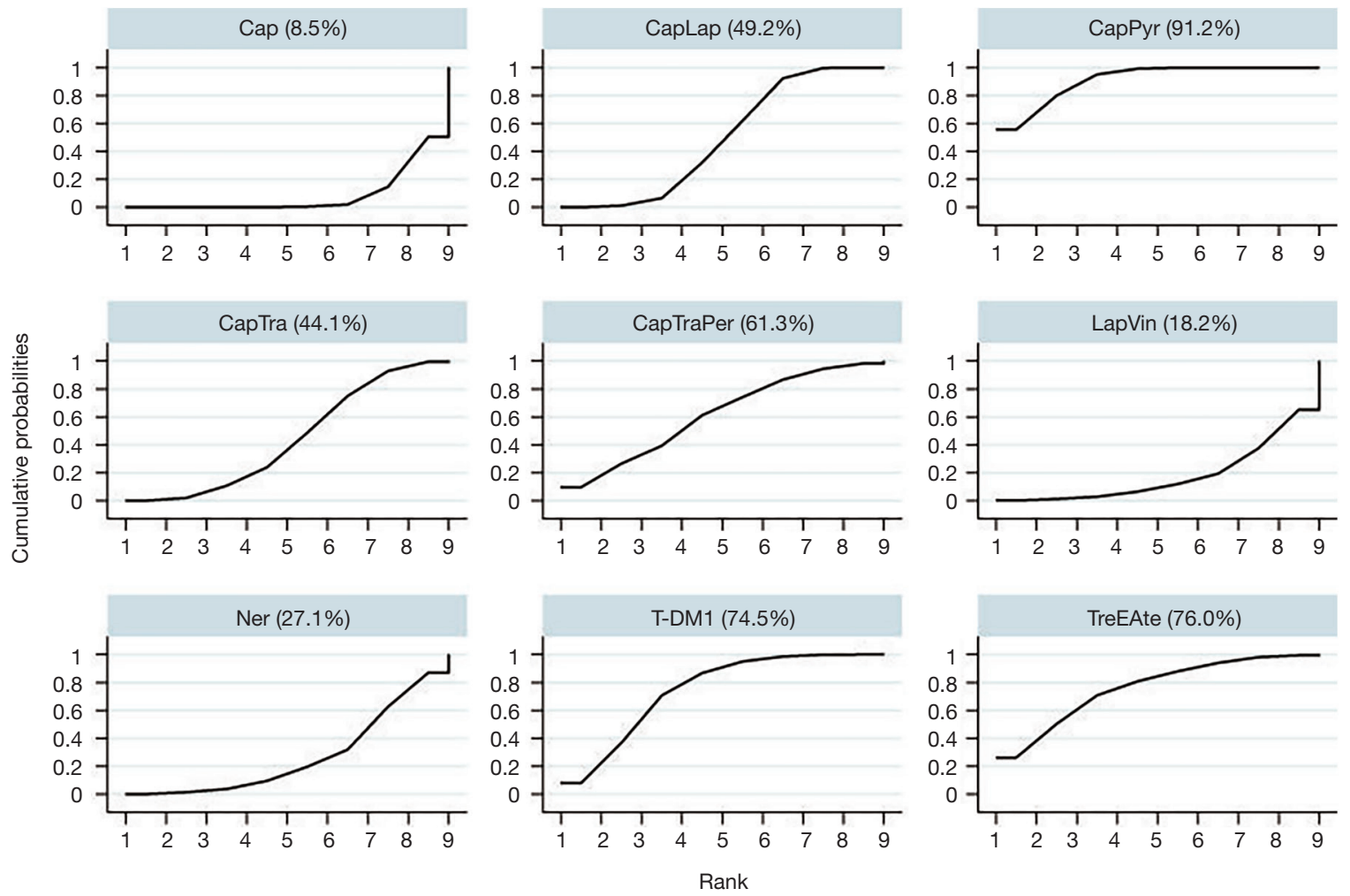

Graphs by treatment

Figure 2 The surface under the cumulative ranking area rank test for overall response rate. Cap, capecitabine; CapTra, capecitabine plus trastuzumab; LapCap, lapatinib plus capecitabine; LapVin, lapatinib plus vinorelbine; Ner, neratinib; PerTraCap, pertuzumab plus trastuzumab plus capecitabine; PyrCap, pyrotinib plus capecitabine; TraE, trastuzumab emtansine; TraEAte, trastuzumab emtansine plus atezolizumab.

not assessed due to insufficient observations. The SUCRA rank showed that SUCRA probabilities of pyrotinib plus capecitabine was $99.1 \%$ which was the highest and it is the most likely treatment to improve PFS for patients with HER2-positive unresectable, locally advanced or metastatic BC after trastuzumab therapy within compared regimens (Figure 3). In pair-wise comparison hazard ratio of pyrotinib plus capecitabine $v s$. other regimens were associated with statistically significant, except for compared with T-DM1 plus atezolizumab (https://cdn.amegroups.cn/static/ public/atm-20-5149-1.xlsx). Hazard ratio of pyrotinib plus capecitabine $v s$. T-DM1 is 0.78 with $95 \%$ CI $(0.69,0.89)$ which is consistent with the SUCRA probabilities result on PFS. The comparison-adjusted funnel plot did not suggest any publication bias (Appendix 2).

\section{OS}

The network plot for OS was presented in Figure S7 of Appendix 2. The loop-specific heterogeneity inconsistency plot is shown in Figure S8 of Appendix 2, and the result of global inconsistency was not assessed due to insufficient observations (Appendix 2). The top 3 SUCRA probability were comparable, which of T-DM1 plus Atezolizumab was $76.1 \%$, pyrotinib plus capecitabine $74.5 \%$ and pertuzumab plus trastuzumab plus capecitabine $71.2 \%$ (Figure 4). There were no significant differences among various HER2targeted regimens for OS by using pair-wise comparisons (https://cdn.amegroups.cn/static/public/atm-20-5149-1. xlsx). The comparison-adjusted funnel plot did not suggest any publication bias (Appendix 2). 

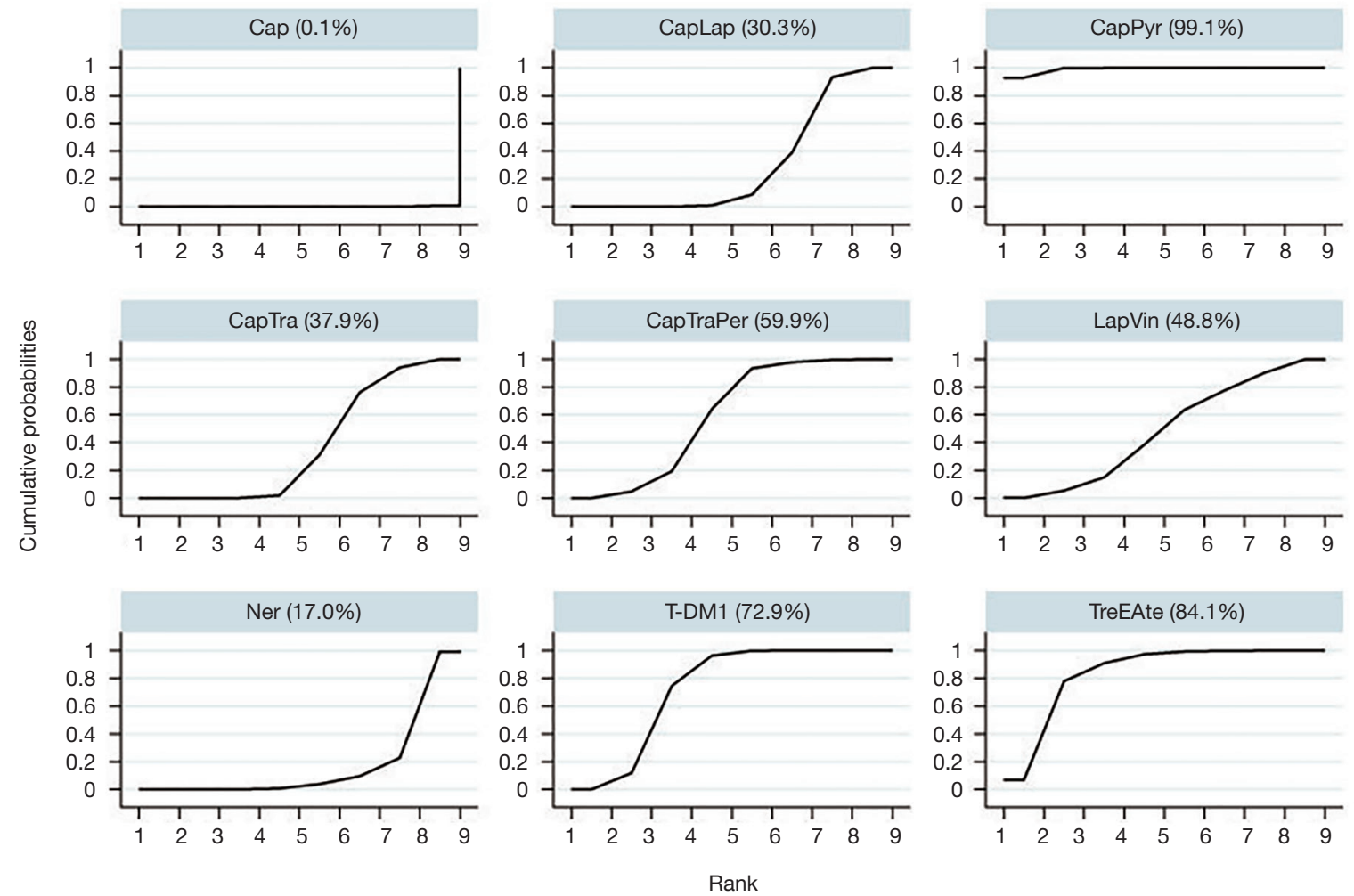

Graphs by treatment

Figure 3 The surface under the cumulative ranking area rank test for progression-free survival. Cap, capecitabine; CapTra, capecitabine plus trastuzumab; LapCap, lapatinib plus capecitabine; LapVin, lapatinib plus vinorelbine; Ner, neratinib; PerTraCap, pertuzumab plus trastuzumab plus capecitabine; PyrCap, pyrotinib plus capecitabine; TraE, trastuzumab emtansine; TraEAte, trastuzumab emtansine plus atezolizumab.

\section{Grade $\geq 3$ adverse events}

Six of included studies reported the incidence of any grade $\geq 3$ adverse events in each group. We noted the incidence of any grade $\geq 3$ adverse events in lapatinib plus capecitabine (353/683), T-DM1 (213/558), trastuzumab plus capecitabine (130/218), pertuzumab plus trastuzumab plus capecitabine (118/228), pyrotinib plus capecitabine (220/384), T-DM1 plus atezolizumab (43/132), and capecitabine (24/94) were $51.7 \%, 38.2 \%, 59.6 \%, 51.8 \%, 57.3 \%, 32.6 \%$, and $25.5 \%$, respectively.

For specific grade $\geq 3$ adverse events, the network metaanalysis showing the comparisons of various HER2targeted regimens on specific grade $\geq 3$ adverse events were presented in Appendix 2. Relatively higher risk (SUCRA $<10$ ) of specific adverse events related to different regimens were as below: The risk of AST increased and thrombocytopenia was highest for patients treated with
T-DM1 plus atezolizumab; patients treated with neratinib and pyrotinib plus capecitabine were associated with higher risk of diarrhea; the risk of neutropenia was highest in patients treated with lapatinib plus vinorelbine. All other details regarding the adverse events $\geq 3$ grade and pair-wise comparison on each regimen were summarized in https:// cdn.amegroups.cn/static/public/atm-20-5149-1.xlsx and Appendix 3. Publication biases for each grade $\geq 3$ adverse events are presented in Appendix 2, and no significant publication bias was detected.

\section{Discussion}

A previous meta-analysis investigated the treatment effect of various HER2-targeted regimens for patients of HER2positive $\mathrm{BC}$ with prior trastuzumab therapy by Paracha et al. (39). It based on 7 RCTs found that T-DM1 was favorable for unresectable, locally advanced or metastatic 

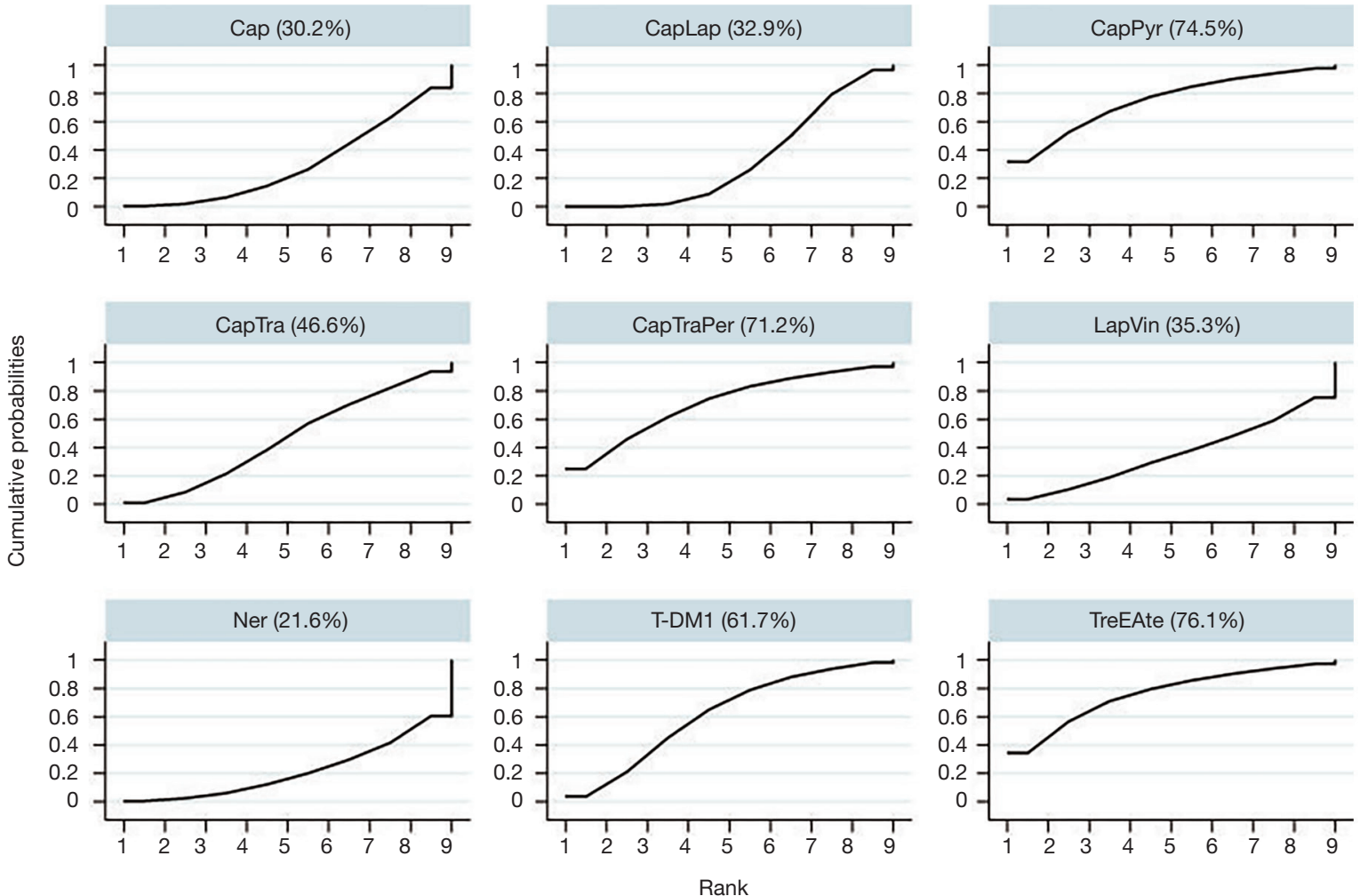

Graphs by treatment

Figure 4 The surface under the cumulative ranking area rank test for overall survival. Cap, capecitabine; CapTra, capecitabine plus trastuzumab; LapCap, lapatinib plus capecitabine; LapVin, lapatinib plus vinorelbine; Ner, neratinib; PerTraCap, pertuzumab plus trastuzumab plus capecitabine; PyrCap, pyrotinib plus capecitabine; TraE, trastuzumab emtansine; TraEAte, trastuzumab emtansine plus atezolizumab.

HER2-positive BC after taxane/trastuzumab treatment (39). However, Paracha et al.'s analysis just included six types of HER2-targeted regimens and several new treatment options such as pyrotinib plus capecitabine and T-DM1 plus Atezolizumab were not included. Our network metaanalysis is innovative for it included current approved and emerging targeted therapies that had reported efficacy results from RCT on HER2-positive BC with prior trastuzumab therapy. This analysis included 12 RCTs and 9 HER2-targeted regimens and it provided a more comprehensive information in this specific population.

In our network meta-analysis, the treatment effects of HER2-targeted regimens on ORR, PFS, OS, and grade $\geq 3$ adverse events in patients with HER2-positive unresectable, locally advanced or metastatic BC after trastuzumab therapy were investigated. This analysis was based on 12 RCTs, and enrolled 3,769 patients randomly assigned to 9 HER2targeted regimens. The result indicated that pyrotinib plus capecitabine had favorable results on ORR and PFS improvement over all other regimens. This superior effect may due to that the irreversible pan-ErbB receptor tyrosine kinase inhibitor, pyrotinib, could to some extent convert trastuzumab resistance after trastuzumab therapy. Evidence have showed that trastuzumab resistance have various mechanisms including p95 HER2 overexpression, MUC4 overexpression, other alterative pathway activation like HER3 and IEGFR and cMET pathway (40). P95 HER2 and MUC4 overexpression prevent trastuzumab from binding to HER2 so that the HER2 pathway downstream could be continuously activated. Pyrotinib binding to the ATP-binding domain of tyrosine kinase which is on the downstream of HER2 pathway can prevent phosphorylation and subsequent activation of the signal transduction pathways, leading to apoptosis and decreasing cellular proliferation $(12,41)$.

With regarding to OS, the SUCRA for T-DM1 plus 
atezolizumab was relatively high among all compared regimens. However T-DM1 plus atezolizumab, pyrotinib plus capecitabine and Pertuzumab plus trastuzumab plus capecitabine had very close probability scores $(76.1 \% v$ s. $74.5 \%$ vs. $71.2 \%$ ). T-DM1 plus atezolizumab is a regimen that is under exploring for its effect on HER2 positive $\mathrm{BC}$ and has not yet been approved and recommended by guidelines. In the KATE2 trial it failed to show superiority over T-DM1 alone although there was a trend that in the PD-L1 positive subgroup T-DM1 plus atezolizumab had numerically higher median PFS than T-DM1 alone (38). The effect of this combination on PD-L1 positive group need to be further explored. Besides T-DM1 plus atezolizumab the SUCRA result indicated that pyrotinib plus capecitabine was more likely to provide OS benefit compared with T-DM1 and all other regimens. In pairwise comparisons all HER2-targeted regimens were not associated with statistically improvement on OS. This could be the reason that only 10 of 12 of included trials reported OS and especially the OS data in several studies including KATE2 and pyrotinib's two phase 3 trials were not mature yet and hadn't reached their median OS.

There was no specific requirement on the treatment duration of previous trastuzumab in metastatic phase but in Emilia study its enrolled population with more strict criteria was within 6 months after treatment for early disease. Generally, the population included in this study contained both primary and secondary trastuzumab resistant patients. These different subgroups may have different reaction on the following anti-HER2 based therapy. In 3 pyrotinib's trials there were further related subgroup analysis which showed that whether patients were resistant to previous trastuzumab therapy they could all gained additional PFS benefit compared to the control arm (23-25). However there was no corresponding subgroup analysis result available in other included trials so we could not do efficacy analysis on primary and secondary trastuzumab resistant subpopulation for all included 12 trials.

It should be noticed that 3 of the included trials contained some patients who didn't received previous trastuzumab. The number were $12(16 \%)$ in experiment arm and 5 (14\%) in control arm of Janni et al. 2014, 2 (5\%) and 2 (5\%) in Takano et al. 2018, 30 (46.2\%) and 29 (46\%) in Ma et al. 2019. However, this population was only $2.2 \%$ $(80 / 3,567)$ of the total analyzed population so its influence on the total result was thought to be limited.

The target population in this analysis is locally advanced and metastatic breast cancer and there was some difference in the constitution of different treatment line patients. Generally, there was a trend that percentage of first line patients was increasing and the second line patients were declining correspondingly with time. This phenomenon was due to the increasing use of trastuzumab in neoadjuvant and adjuvant phase of BC from 2006 to 2020. This population transition may have some effects on this analysis result which could not explore in this analysis.

This analysis focuses on the treatment choices typically on second line in HER2 positive BC with previous trastuzumab therapy. Besides these options discussed above there are quite a few new emerging anti-HER2 targeted agents under exploring in the later line and some of them had reported promising results. Margetuximab is a chimeric anti-HER2 mAb with an Fc domain genetically modified to improve binding to $\mathrm{Fc}$ gamma receptors ( $\mathrm{F} c \gamma \mathrm{Rs}$ ), aiming at increasing ADCC. SOPHIA study showed that the median OS was 21.6 months with margetuximab compared with 19.8 months with trastuzumab (HR 0.89; 95\% CI: 0.69-1.13; $\mathrm{P}=0.33$ ) in the second interim OS analysis (42). In the phase II HER2CLIMB trial tucatinib, an irreversible panHER tyrosine kinase inhibitor, in combination with trastuzumab and capecitabine improved median PFS (7.8 vs. 5.6 m, HR $0.54,95 \%$ CI: 0.42 to $0.71, \mathrm{P}<0.001$ ) and median OS (21.9 vs. $17.4 \mathrm{~m}$, HR 0.66; $95 \%$ CI: 0.50 to $0.88 ; \mathrm{P}=0.005)$ compared to trastuzumab, capecitabine and placebo (43). Trastuzumab-deruxtecan (also known as DS8201) and Trastuzumab-duocarmazine (SYD985) are both antibody-drug conjugates (ADCs) which reported results in heavily pre-treated HER2-positive population. DS8201 showed a response rate of $60.9 \%$ and a median PFS of 16.4 months and $86.2 \%$ of treated patients were alive at 12 months in DESTINY-Breast01 (44). The ORR of SYD985 was $33 \%$, with a median PFS of $7.6 \mathrm{~m}$ in an expansion cohort of 48 HER2 positive heavily pretreated BC (45). Another innovative agent is Zenocutuzumab (MCLA-128), a bispecific humanized full-length IgG1 antibody that binds both HER2 and HER3. In a Phase II trial (NCT03321981) 39 patients who had progressed on trastuzumab/pertuzumab and T-DM1 were treated with zenocutuzumab plus trastuzumab and vinorelbine. The clinical benefit rate (CBR) at 24 weeks (evaluable in 37 patients) was $35.1 \%$ (95\% CI: 22.2-50.0). The ORR was $18.9 \%$ including one patient with a CR (46). With all these emerging evidences several factors should be evaluated for clinician to make a treatment choice in later line treatment for HER2 positive BC. First, previous treatment and related efficacy. Whether part of the previous regimen would be 


\section{Page 10 of 13}

still effective will determine we either apply a totally new drug/combination or keep part of the previous regimen and replace or add another new one. Second, brain metastasis. If patients have brain lesion based on current evidence TKIs combined regimens are recommended. Third, toxicity and quality of life. Different agent has specific characteristics of adverse events which should be considered personally. The last one, convenience. Daily pills to some extent are more convenient. In a word, treatment decision should be based on multiple factors related to both the disease and the patient.

For targeted regimens evaluated in our analysis related grade $\geq 3$ adverse events included hematologic, gastrointestinal, and other nonspecific disorders. Results showed that different type of target agent combined regimen had specific adverse events characteristics. Neratinib and pyrotinib plus capecitabine had higher incidence of diarrhea which were consistent with previous report $(47,48)$ and T-DM1 plus atezolizumab were specifically related with higher incidence of thrombocytopenia and AST increased. Although diarrhea was more common in the treatment with pyrotinib plus capecitabine it could be well managed after being informed with its characteristics ahead. The median onset of diarrhea was 3 days after the use of pyrotinib, and the median duration of diarrhea was 2 days. Most grade $\geq 3$ diarrhea occurred during the first 2 treatment cycles and the median time to occurrence is 11 days and $50 \%$ occurred on day 2 to 15 after starting treatment. The median duration of grade $\geq 3$ diarrhea is 7 days. As study treatment progressed, the incidence of diarrhea overall showed a decreasing trend (23-25). Patient education with diet adjustment and timely symptomatic treatment with loperamide and Montmorillonite Powder is important. Diarrhea related to pyrotinib was generally controllable and seldomly led to treatment interruption (38). Consistent with high incidence of grade $\geq 3$ thrombocytopenia in T-DM1 plus atezolizumab it was reported that T-DM1 treatment alone was also associated with relatively high incidence of thrombocytopenia. In EMILIA study, the incidence of thrombocytopenia was reported is $15 \%$ and especially in the Asian population this incidence is triple as high as $45 \%$ (49). So, this adverse event should be paid more attention and need be monitored during therapy with T-DM1.

The limitations of this study should be acknowledged. First, not all included studies reported the treatment efficacy of various HER2-targeted regimens on OS, and the power potential was not enough to detect significant difference among some of various HER2-targeted regimens in pair-
Li et al. A network meta-analysis for HER2-positive breast cancer

wise comparisons. Second, the result of global inconsistency for each investigated outcome was not evaluated owing to insufficient observations. Third, the results of SUCRA and pair-wise comparisons various HER2-targeted regimens were not completely consistent owing to smaller number of included trials. Forth, publication bias was inevitable because of the analysis of this study based on published or registered data. Finally, the analysis based on study-level, and the detailed analysis regarding patients with specific characteristics were restricted.

Notwithstanding above limitations the results of this analysis provide a more comprehensive and updated evidence to guide the treatment choice regarding HER2targeted regimens for patients with unresectable, locally advanced or metastatic HER2-positive breast cancer after trastuzumab therapy. This study found pyrotinib plus capecitabine could be a better treatment strategy for patients with prior trastuzumab therapy. However, the risks of adverse events such as diarrhea related to it need to be informed and well managed. These results could assist oncologist make better treatment decision for patients with HER2-positive unresectable, locally advanced or metastatic breast cancer after trastuzumab therapy and to bring more benefit to them.

\section{Acknowledgments}

Funding: None.

\section{Footnote}

Reporting Checklist: The authors have completed the PRISMA reporting checklist. Available at http://dx.doi. org/10.21037/atm-20-5149

Conflicts of Interest: All authors have completed the ICMJE uniform disclosure form (available at http://dx.doi. org/10.21037/atm-20-5149). The authors have no conflicts of interest to declare.

Ethical Statement: The authors are accountable for all aspects of the work in ensuring that questions related to the accuracy or integrity of any part of the work are appropriately investigated and resolved.

Open Access Statement: This is an Open Access article distributed in accordance with the Creative Commons Attribution-NonCommercial-NoDerivs 4.0 International 
License (CC BY-NC-ND 4.0), which permits the noncommercial replication and distribution of the article with the strict proviso that no changes or edits are made and the original work is properly cited (including links to both the formal publication through the relevant DOI and the license). See: https://creativecommons.org/licenses/by-nc-nd/4.0/.

\section{References}

1. Momenimovahed Z, Salehiniya H. Epidemiological characteristics of and risk factors for breast cancer in the world. Breast Cancer (Dove Med Press) 2019;11:151-64.

2. Soto-Perez-De-Celis E, Loh KP, Baldini C, et al. Targeted agents for HER2- positive breast cancer in older adults: current and future perspectives. Expert Opin Investig Drugs 2018;27:787-801.

3. Naito Y, Urasaki T. Precision medicine in breast cancer. Chin Clin Oncol 2018;7:29.

4. Miller LD, Chou JA, Black MA, et al. Immunogenic subtypes of breast cancer delineated by gene classifiers of immune responsiveness. Cancer Immunol Res 2016;4:600-10.

5. Hurvitz SA, Dirix L, Kocsis J, et al. Phase II randomized study of trastuzumab emtansine versus trastuzumab plus docetaxel in patients with human epidermal growth factor receptor-2 positive metastatic breast cancer. J Clin Oncol 2013;31:1157-63.

6. Cronin KA, Harlan LC, Dodd KW, et al. Populationbased estimate of the prevalence of HER-2 positive breast cancer tumors for early stage patients in the US. Cancer Invest 2010;28:963-8.

7. Ricci F, Le Tourneau C. Trastuzumab emtansine in HER2-positive metastatic breast cancer: what is the best sequence? Chin Clin Oncol 2018;7:3.

8. Slamon DJ, Leyland-Jones B, Shak S, et al. Use of chemotherapy plus a monoclonal antibody against HER2 for metastatic breast cancer that overexpresses HER2. N Engl J Med 2001;344:783-92.

9. Marty M, Cognetti F, Maraninchi D, et al. Randomized phase II trial of the efficacy and safety of trastuzumab combined with docetaxel in patients with human epidermal growth factor receptor 2-positive metastatic breast cancer administered as first-line treatment: the M77001 study group. J Clin Oncol 2005;23:4265-74.

10. Baselga J, Cortés J, Kim SB. Pertuzumab plus trastuzumab plus docetaxel for metastatic breast cancer. N Engl J Med 2012;366:109-19.

11. Dawood S, Broglio K, Buzdar AU. Prognosis of women with metastatic breast cancer by HER2 status and trastuzumab treatment: an institutional-based review. J Clin Oncol 2010;28:92-8

12. Narayan M, Wilken JA, Harris LN, et al. Trastuzumabinduced HER reprogramming in "resistant" breast carcinoma cells. Cancer Res 2009;69:2191-4.

13. Baselga J, Cortes J, Im SA, et al. Biomarker analyses in CLEOPATRA: a phase III, placebo-controlled study of pertuzumab in human epidermal growth factor receptor 2-positive, first-line metastatic breast cancer. J Clin Oncol 2014;32:3753-61.

14. Harbeck N, Beckmann MW, Rody A, et al. HER2 dimerization inhibitor pertuzumab-mode of action and clinical data in breast cancer. Breast Care (Basel) 2013;8:49-55.

15. Baselga J, Cortes J, Kim SB, et al. Pertuzumab plus trastuzumab plus docetaxel for metastatic breast cancer. $\mathrm{N}$ Engl J Med 2012;366:109-19.

16. Blackwell KL, Burstein HJ, Storniolo AM, et al. Randomized study of lapatinib alone or in combination with trastuzumab in women with ErbB2-positive, trastuzumab-refractory metastatic breast cancer. J Clin Oncol 2010;28:1124-30.

17. von Minckwitz G, du Bois A, Schmidt M, et al. Trastuzumab beyond progression in human epidermal growth factor receptor 2-positive advanced breast cancer: a German breast group 26/breast international group 03-05 study. J Clin Oncol 2009;27:1999-2006.

18. Extra JM, Antoine EC, Vincent-Salomon A, et al. Efficacy of trastuzumab in routine clinical practice and after progression for metastatic breast cancer patients: the observational Hermine study. Oncologist 2010;15:799-809.

19. Baselga J, Javier Cortés J, Kim SB, et al. Pertuzumab plus trastuzumab plus docetaxel for metastatic breast cancer. $\mathrm{N}$ Engl J Med 2012;366:109-19.

20. Gamucci T, Pizzuti L, Natoli C, et al. A multicenter REtrospective observational study of first-line treatment with PERtuzumab, trastuzumab and taxanes for advanced HER2 positive breast cancer patients. RePer Study. Cancer Biol Ther. 2019;20:192-200.

21. Geyer CE, Forster J, Lindquist D, et al. Lapatinib plus capecitabine for HER2-positive advanced breast cancer. N Engl J Med 2006;355:2733-43.

22. Verma S, Miles D, Gianni L, et al. Trastuzumab emtansine for HER2-positive advanced breast cancer. N Engl J Med 2012;367:1783-91.

23. Ma F, Ouyang Q, Li W, et al. Pyrotinib or Lapatinib 


\section{Page 12 of 13}

Combined with Capecitabine in HER2-Positive Metastatic Breast Cancer with Prior Taxanes, Anthracyclines, and/ or Trastuzumab: A Randomized, Phase II Study. J Clin Oncol 2019;37:2610-9.

24. Yan M, Bian Li, Hu X, et al. Pyrotinib plus capecitabine for human epidermal growth factor receptor 2-positive metastatic breast cancer after trastuzumab and taxanes (PHENIX): a randomized, double-blind, placebocontrolled phase 3 study. Transl Breast Cancer Res 2020;1:13.

25. Yan $M$, Ma F, Hu X, et al. pyrotinib or lapatinib plus capecitabine for HER2 + metastatic breast cancer (Pheobe): a randomized phase 3 trial. 2020 ASCO, Abstract 1003.

26. Higgins JP, Welton NJ. Network meta-analysis: a norm for comparative effectiveness? Lancet 2015;386:628-30.

27. Moher D, Liberati A, Tetzlaff J, et al. Preferred Reporting Items for Systematic Reviews and Meta-Analyses: The PRISMA Statement. Plos Med 2009;6:e1000097.

28. Jadad AR, Moore RA, Carroll D. Assessing the quality of reports of randomized clinical trials: is blinding necessary? Control Clin Trials 1996;17:1-12.

29. White IR, Barrett JK, Jackson D, et al. Consistency and inconsistency in network meta-analysis: model estimation using multivariate meta-regression. Res Synth Methods 2012;3:111-25.

30. Bucher HC, Guyatt GH, Griffith LE, et al. The results of direct and indirect treatment comparisons in metaanalysis of randomized controlled trials. J Clin Epidemiol 1997;50:683-91.

31. Salanti G, Ades AE, Ioannidis JP. Graphical methods and numerical summaries for presenting results from multipletreatment meta-analysis: an overview and tutorial. J Clin Epidemiol 2011;64:163-71.

32. Trinquart L, Chatellier G, Ravaud P. Adjustment for reporting bias in network meta-analysis of antidepressant trials. BMC Med Res Methodol 2012;12:150.

33. Martin M, Bonneterre J, Geyer CE Jr, et al. A phase two randomized trial of neratinib monotherapy versus lapatinib plus capecitabine combination therapy in patients with HER2 + advanced breast cancer. Eur J Cancer 2013;49:3763-72.

34. Janni W, Sarosiek T, Karaszewska B, et al. A phase II, randomized, multicenter study evaluating the combination of lapatinib and vinorelbine in women with ErbB2 overexpressing metastatic breast cancer. Breast Cancer Res Treat 2014;143:493-505.

35. Pivot X, Manikhas A, Żurawski B, et al. CEREBEL (EGF111438): A Phase III, Randomized, Open-Label Study of Lapatinib Plus Capecitabine Versus Trastuzumab

\section{Li et al. A network meta-analysis for HER2-positive breast cancer}

Plus Capecitabine in Patients with Human Epidermal Growth Factor Receptor 2-Positive Metastatic Breast Cancer. J Clin Oncol 2015;33:1564-73.

36. Urruticoechea A, Rizwanullah M, Im SA, et al. Randomized phase III trial of trastuzumab plus capecitabine with or without pertuzumab in patients with human epidermal growth factor receptor 2-positive metastatic breast cancer who experienced disease progression during or after trastuzumab-based therapy. J Clin Oncol 2017;35:3030-8.

37. Takano T, Tsurutani J, Takahashi M, et al. A randomized phase II trial of trastuzumab plus capecitabine versus lapatinib plus capecitabine in patients with HER2positive metastatic breast cancer previously treated with trastuzumab and taxanes: WJOG6110B/ELTOP. Breast 2018;40:67-75.

38. Emens LA, Francisco J, Esteva FJ, et al. Trastuzumab emtansine plus atezolizumab versus trastuzumab emtansine plus placebo in previously treated, HER2positive advanced breast cancer (KATE2): a phase 2, multicentre, randomised, double-blind trial. Lancet Oncol 2020;21:1283-95.

39. Paracha N, Reyes A, Diéras V, et al. Evaluating the clinical effectiveness and safety of various HER2-targeted regimens after prior taxane/trastuzumab in patients with previously treated, unresectable, or metastatic HER2positive breast cancer: a systematic review and network meta-analysis. Breast Cancer Res Treat 2020;180:597-609.

40. Pohlmann PR, Mayer IA, Ray Mernaugh R. Resistance to Trastuzumab in Breast Cancer. Clin Cancer Res 2009;15:7479-91.

41. Li X, Yang C, Wan H, et al. Discovery and development of pyrotinib: A novel irreversible EGFR/HER2 dual tyrosine kinase inhibitor with favorable safety profiles for the treatment of breast cancer. Eur J Pharm Sci 2017;110:51-61.

42. Rugo HS, Im SA, Cardoso F, et al. Phase 3 SOPHIA study of margetuximab + chemotherapy vs trastuzumab + chemotherapy in patients with HER2 + metastatic breast cancer after prior anti-HER2 therapies: second interim overall survival analysis. Presented at: San Antonio Breast Cancer Symposium SABCS 2019, abstract GS1-02.

43. Murthy RK, Loi S, Okines A, et al. Tucatinib, Trastuzumab, and Capecitabine for HER2-Positive Metastatic Breast Cancer. N Engl J Med 2020;382:597-609.

44. Modi S, Saura C, Yamashita T, et al. Trastuzumab Deruxtecan in previously treated HER2-positive breast cancer. N Engl J Med 2020;382:610-21. 
45. Banerji U, van Herpen CML, Saura C, et al, Trastuzumab duocarmazine in locally advanced and metastatic solid tumours and HER2-expressing breast cancer: a phase 1 dose-escalation and dose-expansion study. Lancet Oncol 2019;20:1124-35.

46. Hamilton EP, Petit T, Pistilli B, et al. Clinical activity of MCLA-128 (zenocutuzumab), trastuzumab, and vinorelbine in HER2 amplified metastatic breast cancer (MBC) patients (pts) who had progressed on anti-HER2 ADCs. Presented at the ASCO Annual Meeting 2020, abstract 3093.

47. Ma F. Li Q, Chen S, et al. Phase I Study and Biomarker Analysis of Pyrotinib, a Novel Irreversible Pan-ErbB Receptor Tyrosine Kinase Inhibitor, in Patients with

Cite this article as: $\mathrm{Li} \mathrm{X}, \mathrm{Wu} \mathrm{S}$, Zhang L, Zhu J, Xu B. HER2targeted regimens after prior trastuzumab for patients with HER2-positive unresectable, locally advanced or metastatic breast cancer: a network meta-analysis of randomized controlled trials. Ann Transl Med 2020;8(24):1634. doi: 10.21037/atm-205149
Human Epidermal Growth Factor Receptor 2-Positive Metastatic Breast Cancer. J Clin Oncol 2017;35:3105-12.

48. Saura C. Oliveira M, Feng YH, et al. Neratinib Plus Capecitabine Versus Lapatinib Plus Capecitabine in HER2-Positive Metastatic Breast Cancer Previously Treated With $\geq 2$ HER2-Directed Regimens: Phase III NALA Trial. J Clin Oncol 2020;38:3138-49.

49. Diéras V, Miles D, Verma S, et al. Trastuzumab emtansine versus capecitabine plus lapatinib in patients with previously treated HER2-positive advanced breast cancer (EMILIA): a descriptive analysis of final overall survival results from a randomised, open-label, phase 3 trial. Lancet Oncol 2017;18:732-42. 


\section{Appendix 1 Search strategies in electronic databases}

\section{Search strategies in PubMed and Cochrane library}

(1) MeSH descriptor Breast Neoplasms explode all trees

(2) MeSH descriptor Breast explode all trees

(3) breast

(4) (\#1 OR \#2 OR \#3)

(5) MeSH descriptor Neoplasm Metastasis explode all trees

(6) (advanced OR metastat* OR refract* OR recurren* OR salva* OR (late adj stage) OR resistan* OR "stage III" OR (stage AND III*) OR "stage IV" OR "stage 3" OR "stage 4" OR "stage IIIC" OR "stage IIIB" OR unresect*)

(7) $\mathrm{MeSH}$ descriptor Neoplasm Recurrence, Local explode all trees

(8) (\#5 OR \#6 OR \#7)

(9) MeSH descriptor Receptor, Epidermal Growth Factor explode all trees

(10) MeSH descriptor Receptor, erbB-2 explode all trees

(11) HER2 OR HER-2 OR "HER 2" OR "HER positive" OR HER-positive OR HER-overexpressing OR "HER overexpressing" OR ErbB2 OR Erb-B2 OR "Human epidermal growth factor receptor 2" OR "cerbB 2" OR Her2neu

(12) (\#9 OR \#10 OR \#11)

(13) Second-line OR "Second line" OR Secondary OR Progress* OR Relapse* OR Recurren* OR Fail* OR Resist* OR Pretreated OR "pre treated" OR "pre-treated" OR Refract* OR Salvage OR Prior OR Previous*

(14) MeSH descriptor Antineoplastic Protocols explode all trees

(15) MeSH descriptor Drug Therapy explode all trees

(16) (Biologic* OR chemo* OR systemic OR target*) NEAR/2 (therap* OR treat*)

(17) "antineoplastic agent" OR antineoplastic OR antitumor OR anticancer OR "tumor inhibitor" OR "anti tumor" OR "anti tumour" OR antitumour

(18) "angiogenesis inhibitor" OR "neovascularization inhibitor" OR "monoclonal antibody"

(19) "her2 inhibitor" OR "her 2 inhibitor" OR "angiogen inhibitor" OR "her2 antibody" OR "her 2 antibody" OR "ErbB2 inhibitor" OR "ErbB2 antibody"

(20) OR/14-19

(21) $\mathrm{AND} / 4,8,12,13,20$

\section{EmBASE:}

[1] 'chemotherapy'/exp OR chemotherap* OR (chemo* OR biologic* OR hormon* OR endocrin* OR target*) NEAR/2 (therap* OR treat*)

[2] 'antineoplastic agent'/syn OR 'antineoplastic drug' OR 'anticancer drug' OR 'tumor inhibitor' OR 'anticarcinogenic agents' OR 'anticarcinogenic agents'

[3] 'biological therapy'/syn OR 'biologic therapy' OR 'tissue therapy'

[4] 'systemic therapy'/syn

[5] 'molecularly targeted therapy'/syn

[6] 'hormonal therapy'/syn OR 'endocrine therapy' OR 'endocrine treatment'

[7] 'angiogenesis inhibitor'/syn

[8] 'aromatase inhibitor'/syn

[9] ((her2 OR 'her 2' OR 'her 2' OR angiogen*) NEAR/4 inhibit*) OR antiangiogen* OR 'anti angiogen' OR ('her 2' OR her2 OR erbb2 OR 'erbb 2') NEXT/2 antibody

[10] OR/1-9

[11] 'breast tumor'/exp OR 'breast tumour' OR 'breast tumor'

[12] 'breast'/exp OR 'breast'

[13] 'breast neoplasms'/exp OR 'breast neoplasm' OR breast NEAR/5 carcinoma OR breast NEAR/5 cancer OR breast 
NEAR/5 malignan*

[14] OR/11-13

[15] advanced OR metastat* OR refract* OR recurren* OR salva* OR 'late' NEXT/2 'stage' OR resistan* OR 'stage iii' OR (stage AND iii*) OR 'stage iv' OR 'stage 3' OR 'stage 4' OR 'breast metastasis'/exp OR 'metastasis'/exp OR 'recurrent disease'/exp

[16] 'epidermal growth factor receptor 2'/syn OR her2 OR 'her 2' OR 'her-2'

[17] second* OR progress* OR relapse* OR recurren* OR fail* OR resistance OR pretreated OR 'pre treated' OR 'pretreated' OR refract* OR previous* OR salvage OR prior OR 'second-line' OR 'second line'

[18] AND/10,14,15,16,17 


\section{Appendix 2 Network of comparisons and funnel plots for investigated outcomes}

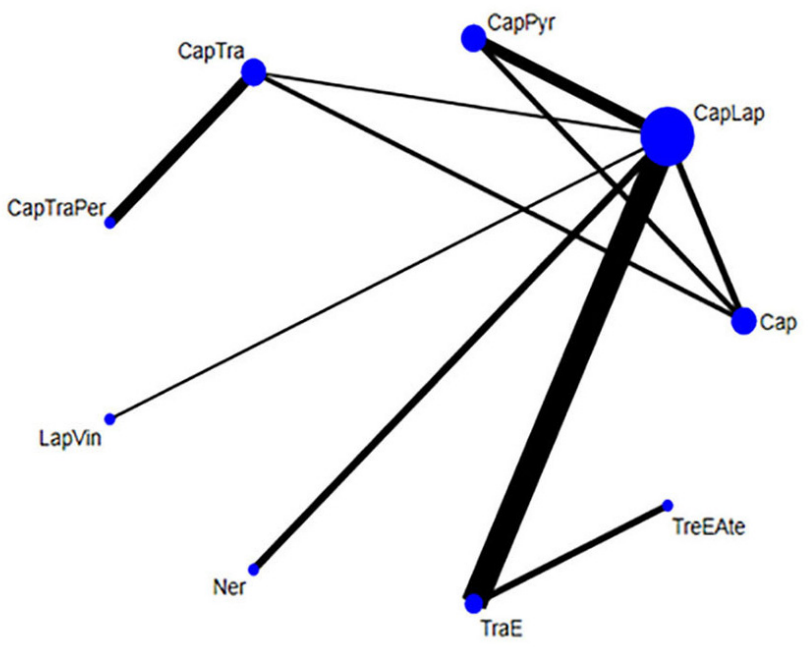

Figure S1 Network of comparisons for overall response rate included in the analysis. Cap, capecitabine; CapTra, capecitabine plus trastuzumab; LapCap, lapatinib plus capecitabine; LapVin, lapatinib plus vinorelbine; Ner, neratinib; PerTraCap, pertuzumab plus trastuzumab plus capecitabine; PyrCap, pyrotinib plus capecitabine; TRAE, trastuzumab emtansine; TraEAte, trastuzumab emtansine plus atezolizumab.

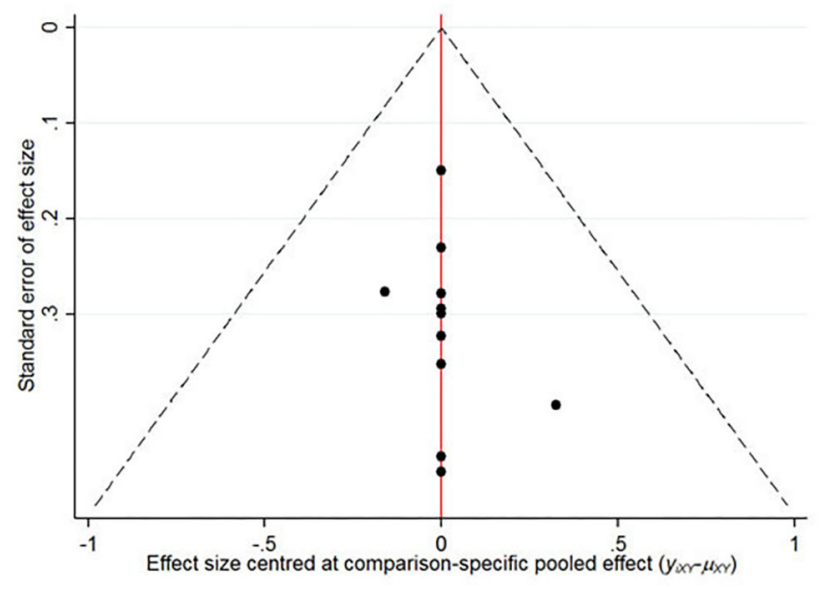

Figure S3 Funnel plot for overall response rate.
$95 \% \mathrm{Cl}$

IF (truncated)

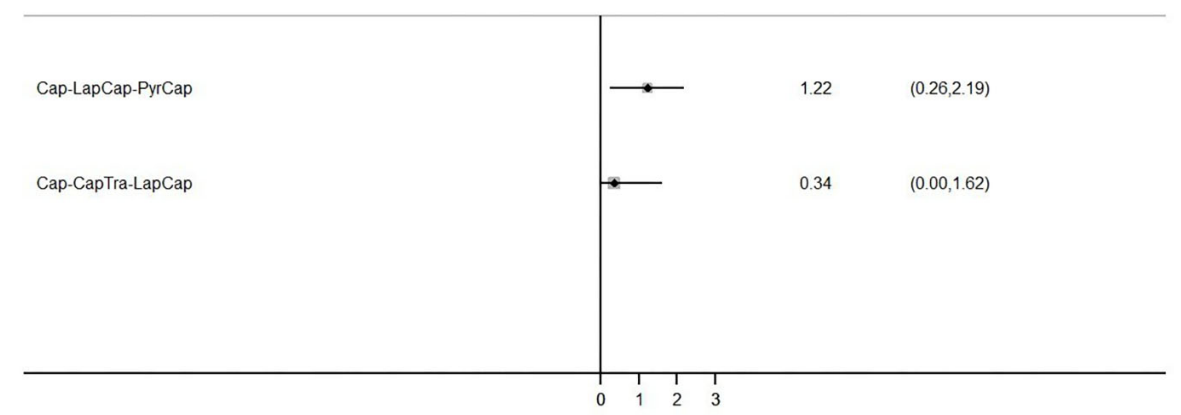

Figure S2 Inconsistency plot for overall response rate. 


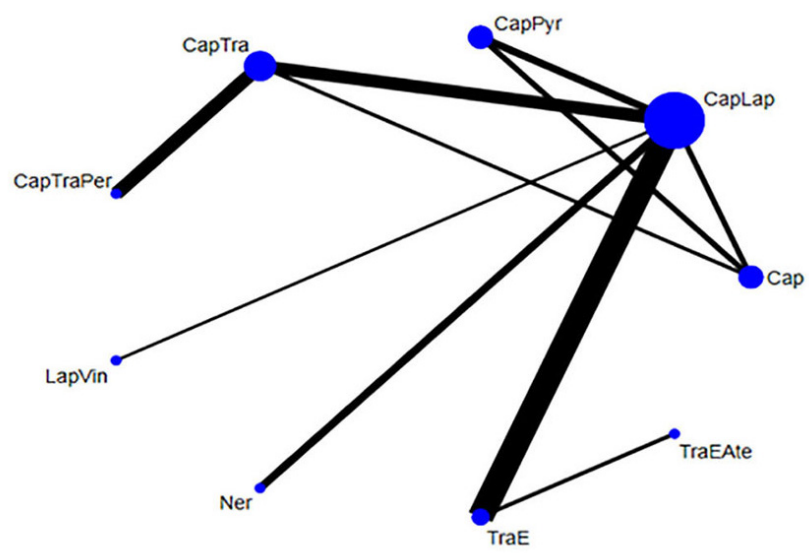

Figure S4 Network of comparisons for progression-free survival included in the analysis. Cap, capecitabine; CapTra, capecitabine plus trastuzumab; LapCap, lapatinib plus capecitabine; LapVin, lapatinib plus vinorelbine; Ner, neratinib; PerTraCap, pertuzumab plus trastuzumab plus capecitabine; PyrCap, pyrotinib plus capecitabine; TRAE, trastuzumab emtansine; TraEAte, trastuzumab emtansine plus atezolizumab.

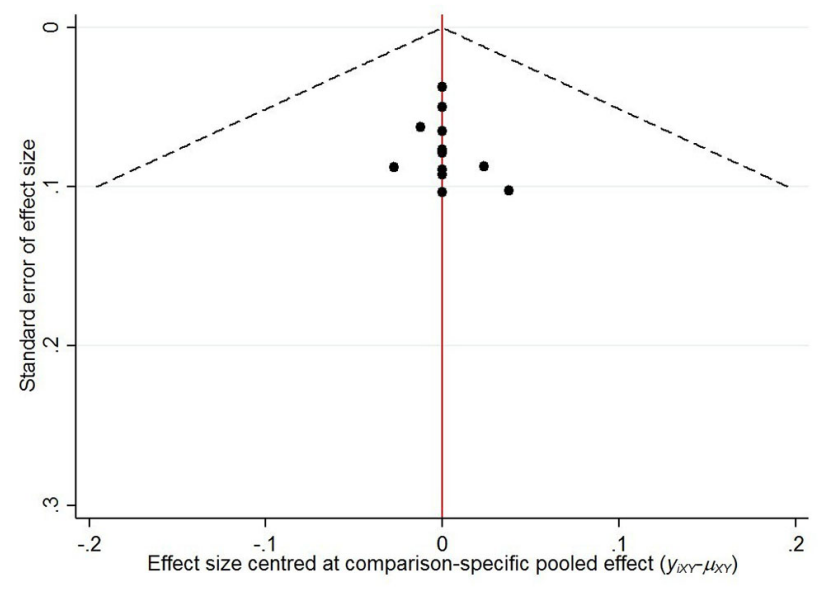

Figure S6 Funnel plot for progression-free survival.

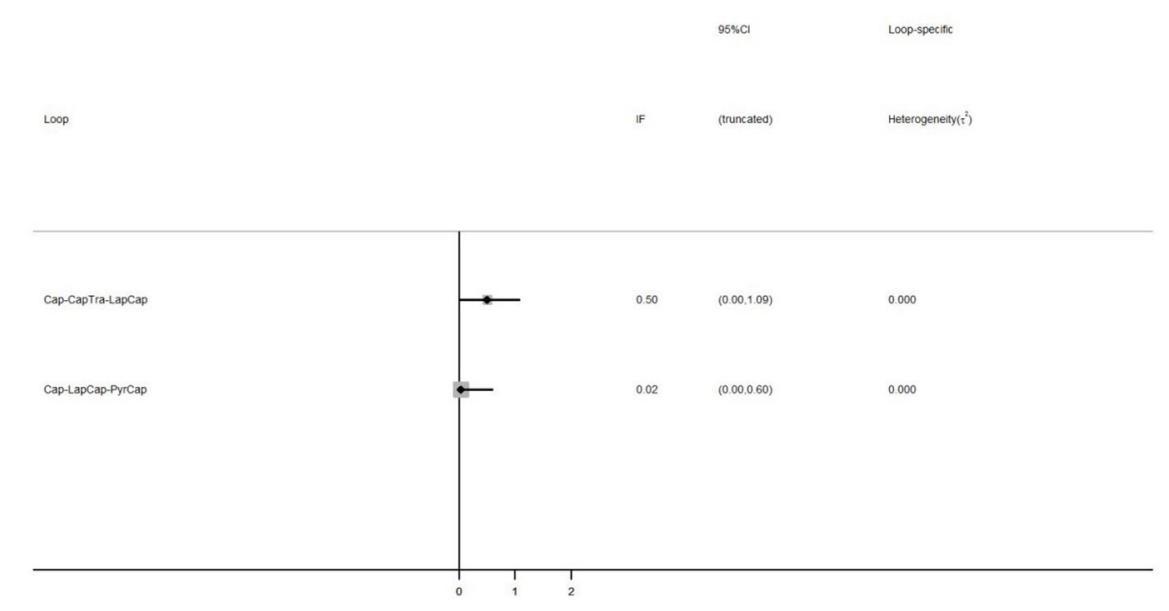

Figure S5 Inconsistency plot for progression-free survival. 


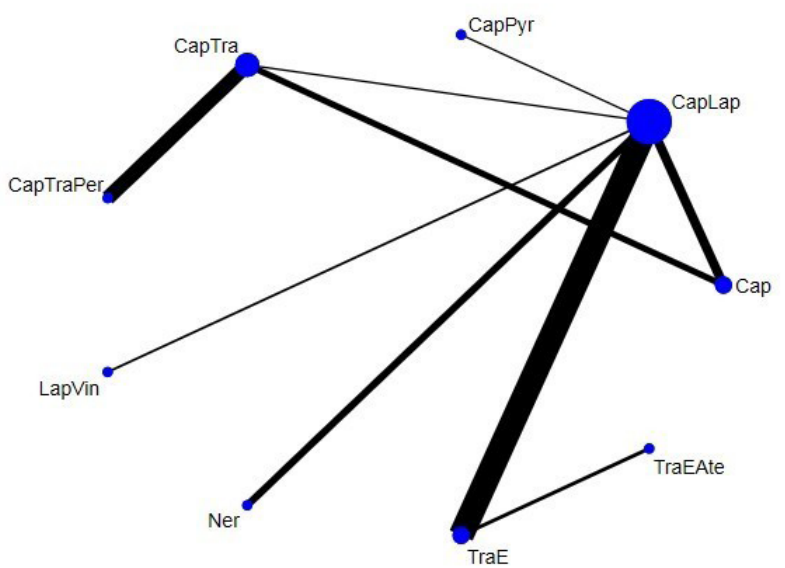

Figure S7 Network of comparisons for overall survival included in the analysis. Cap, capecitabine; CapTra, capecitabine plus trastuzumab; LapCap, lapatinib plus capecitabine; LapVin, lapatinib plus vinorelbine; Ner, neratinib; PerTraCap, pertuzumab plus trastuzumab plus capecitabine; PyrCap, pyrotinib plus capecitabine; TRAE, trastuzumab emtansine; TraEAte, trastuzumab emtansine plus atezolizumab.

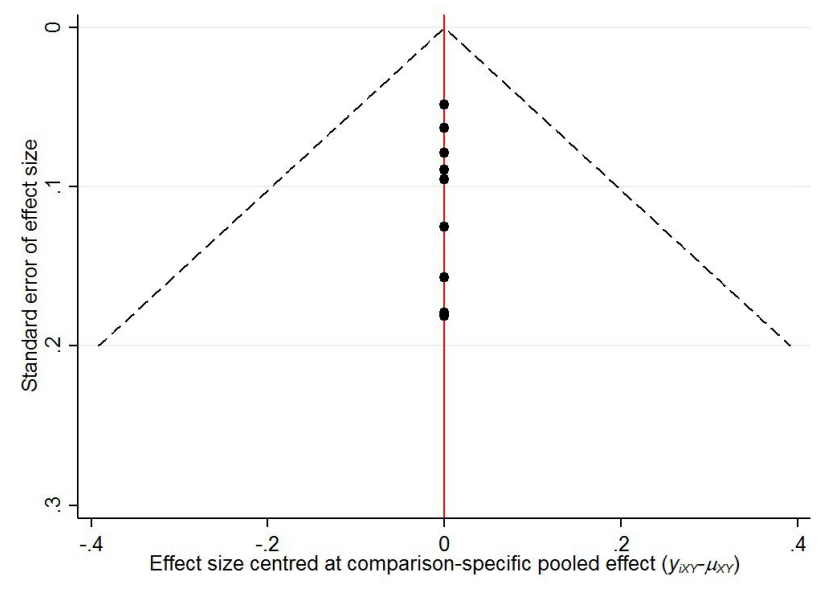

Figure S9 Funnel plot for overall survival.
95\%Cl Loop-specific

Heterogeneity (I')
IF
(truncated)
Loop (truncated)

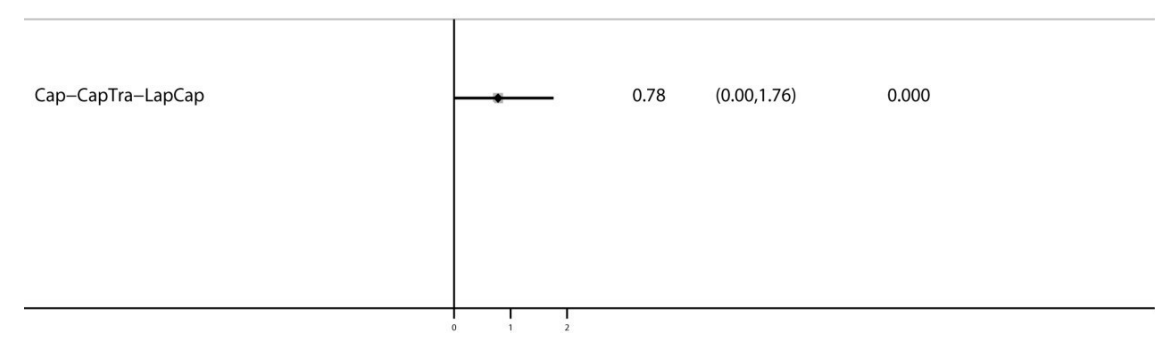

Figure S8 Inconsistency plot for overall survival. 


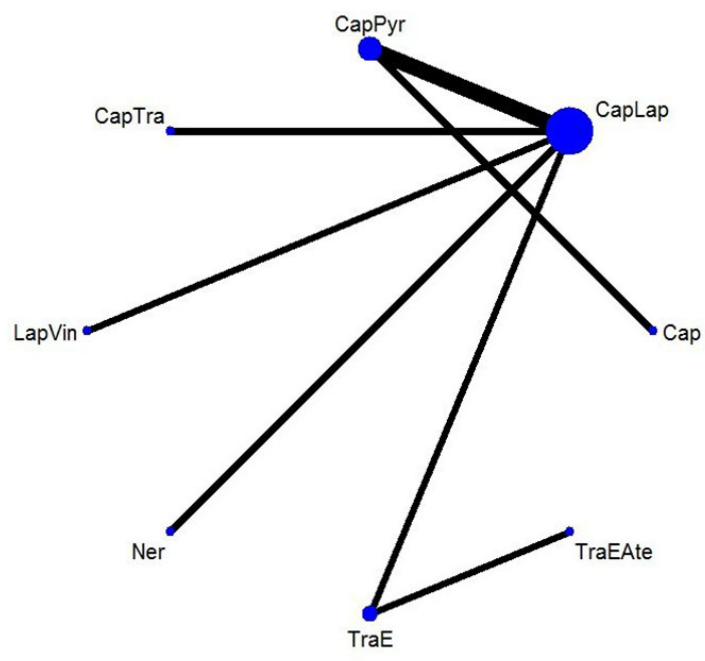

Figure S10 Network of comparisons for alanine transaminase increased included in the analysis. Cap, capecitabine; CapTra, capecitabine plus trastuzumab; LapCap, lapatinib plus capecitabine; LapVin, lapatinib plus vinorelbine; Ner, neratinib; PyrCap, pyrotinib plus capecitabine; TRAE, trastuzumab emtansine; TraEAte, trastuzumab emtansine plus atezolizumab.

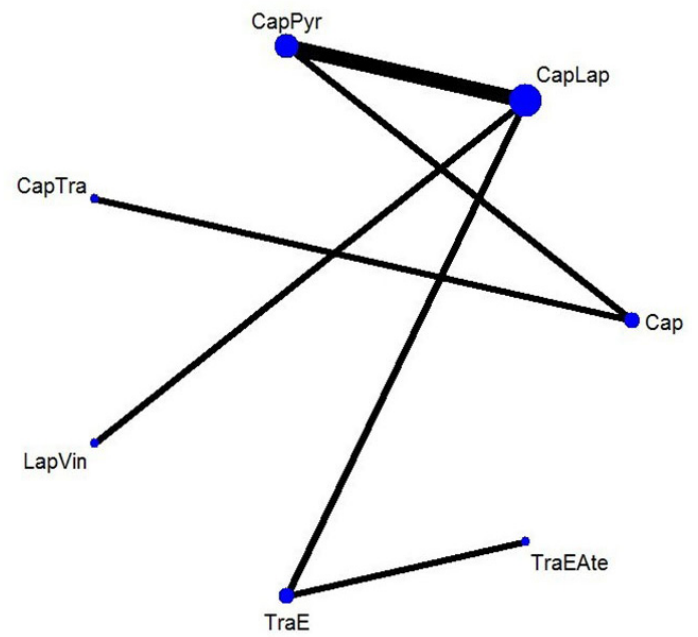

Figure S11 Network of comparisons for anemia included in the analysis. Cap, capecitabine; CapTra, capecitabine plus trastuzumab; LapCap, lapatinib plus capecitabine; LapVin, lapatinib plus vinorelbine; Ner, neratinib; PyrCap, pyrotinib plus capecitabine; TRAE, trastuzumab emtansine; TraEAte, trastuzumab emtansine plus atezolizumab.

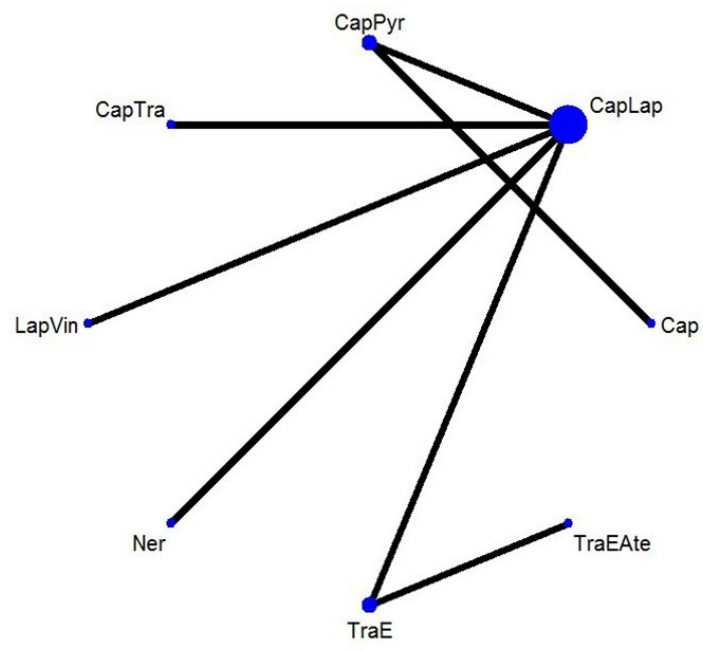

Figure S12 Network of comparisons for aspartate aminotransferase increased included in the analysis. Cap, capecitabine; CapTra, capecitabine plus trastuzumab; LapCap, lapatinib plus capecitabine; LapVin, lapatinib plus vinorelbine; Ner, neratinib; PyrCap, pyrotinib plus capecitabine; TRAE, trastuzumab emtansine; TraEAte, trastuzumab emtansine plus atezolizumab.

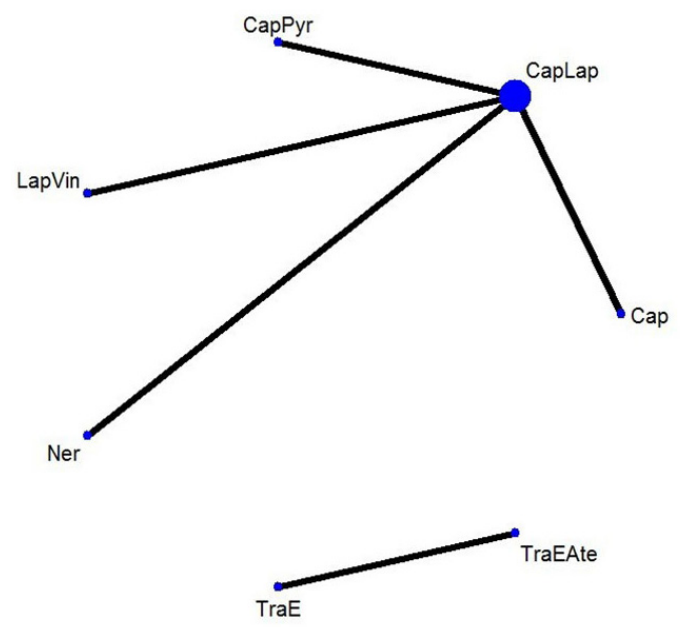

Figure S13 Network of comparisons for asthenia included in the analysis. Cap, capecitabine; LapCap, lapatinib plus capecitabine; LapVin, lapatinib plus vinorelbine; Ner, neratinib; PyrCap, pyrotinib plus capecitabine; TraEAte, trastuzumab emtansine plus atezolizumab. 


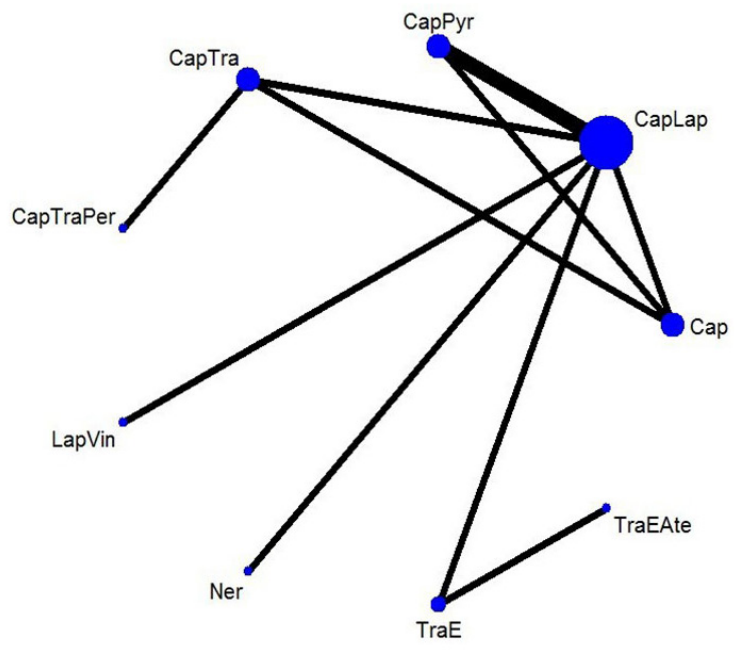

Figure S14 Network of comparisons for diarrhea included in the analysis. Cap, capecitabine; CapTra, capecitabine plus trastuzumab; LapCap, lapatinib plus capecitabine; LapVin, lapatinib plus vinorelbine; Ner, neratinib; PerTraCap, pertuzumab plus trastuzumab plus capecitabine; PyrCap, pyrotinib plus capecitabine; TRAE, trastuzumab emtansine; TraEAte, trastuzumab emtansine plus atezolizumab.

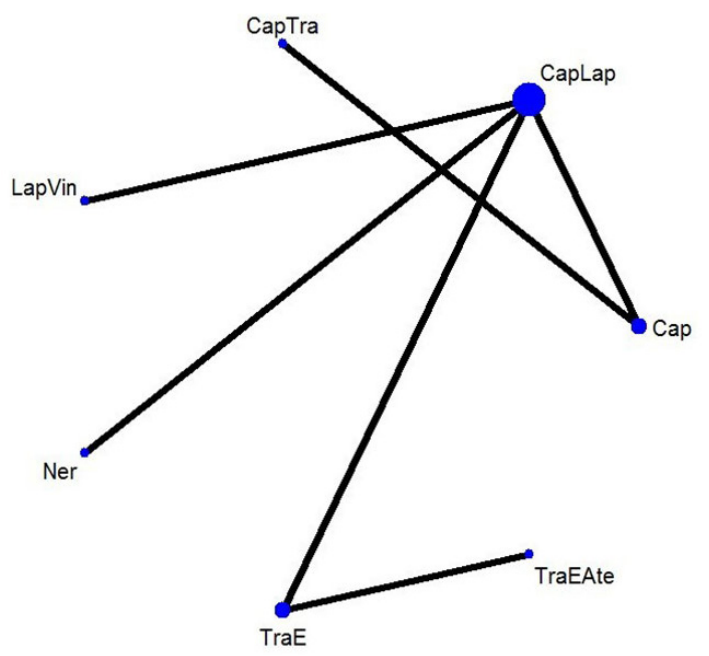

Figure S15 Network of comparisons for fatigue included in the analysis. Cap, capecitabine; CapTra, capecitabine plus trastuzumab; LapCap, lapatinib plus capecitabine; LapVin, lapatinib plus vinorelbine; Ner, neratinib; TRAE, trastuzumab emtansine; TraEAte, trastuzumab emtansine plus atezolizumab.

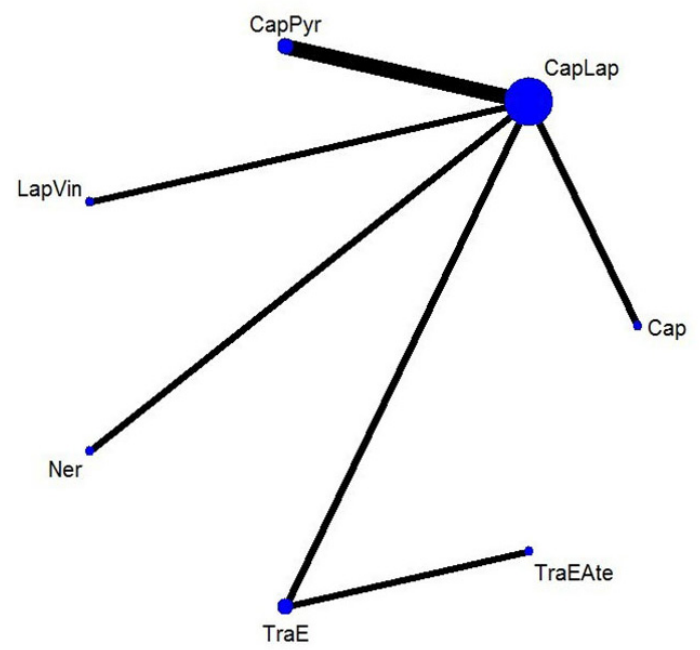

Figure S16 Network of comparisons for nausea included in the analysis. Cap, capecitabine; LapCap, lapatinib plus capecitabine; LapVin, lapatinib plus vinorelbine; Ner, neratinib; PyrCap, pyrotinib plus capecitabine; TRAE, trastuzumab emtansine; TraEAte, trastuzumab emtansine plus atezolizumab

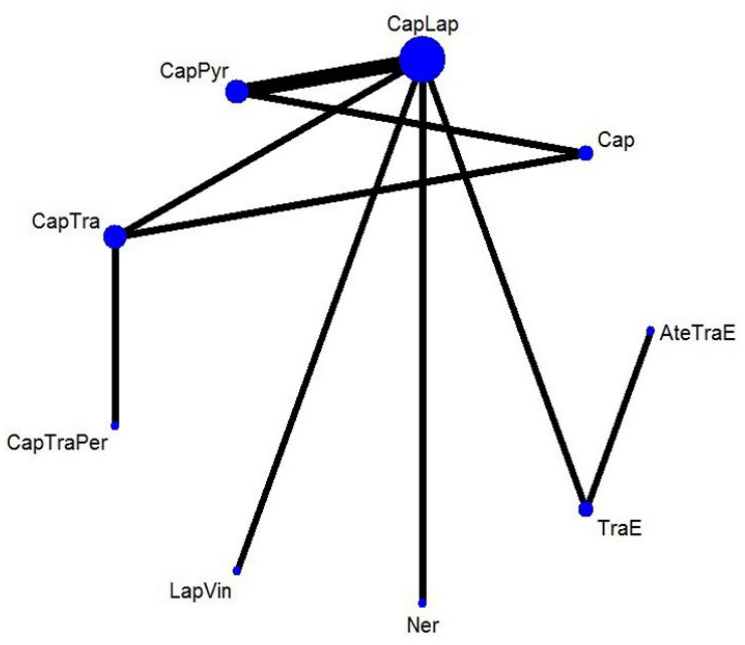

Figure S17 Network of comparisons for neutropenia included in the analysis. Cap, capecitabine; CapTra, capecitabine plus trastuzumab; LapCap, lapatinib plus capecitabine; LapVin, lapatinib plus vinorelbine; Ner, neratinib; PerTraCap, pertuzumab plus trastuzumab plus capecitabine; PyrCap, pyrotinib plus capecitabine; TRAE, trastuzumab emtansine; TraEAte, trastuzumab emtansine plus atezolizumab. 


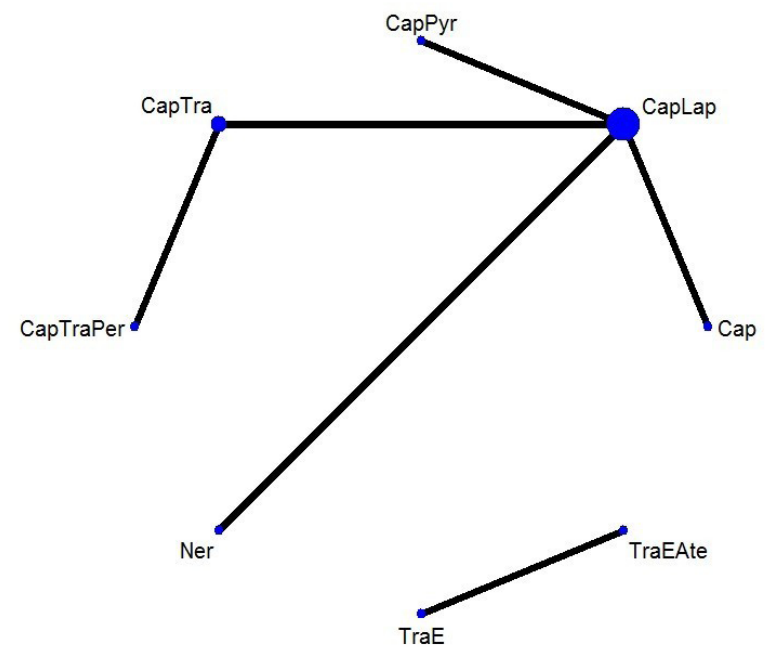

Figure S18 Network of comparisons for rash included in the analysis. Cap, capecitabine; CapTra, capecitabine plus trastuzumab; LapCap, lapatinib plus capecitabine; Ner, neratinib; PerTraCap, pertuzumab plus trastuzumab plus capecitabine; PyrCap, pyrotinib plus capecitabine; TraEAte, trastuzumab emtansine plus atezolizumab.

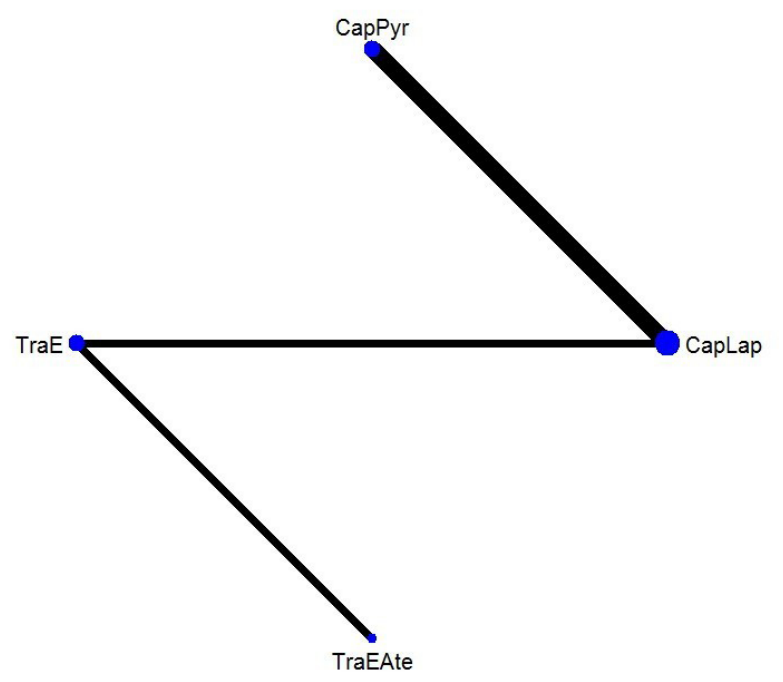

Figure S19 Network of comparisons for thrombocytopenia included in the analysis. LapCap, lapatinib plus capecitabine; PyrCap, pyrotinib plus capecitabine; TRAE, trastuzumab emtansine; TraEAte, trastuzumab emtansine plus atezolizumab.

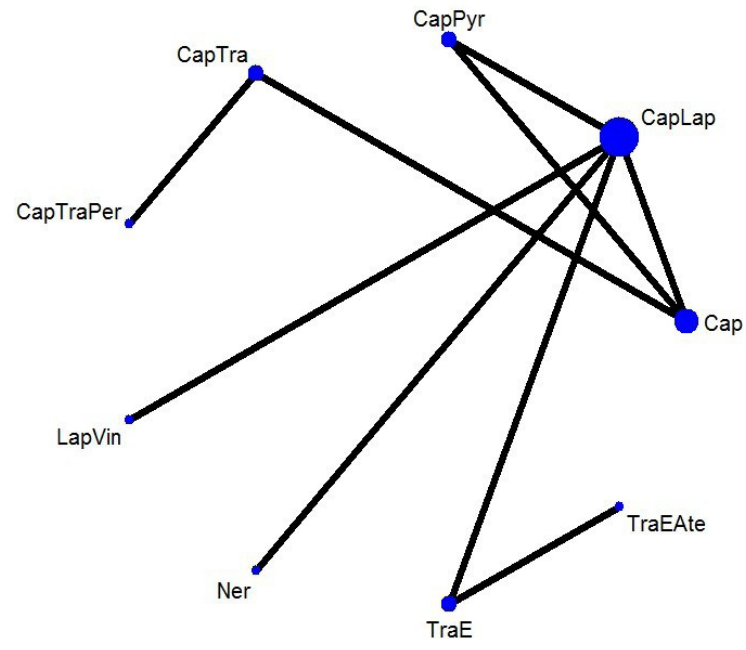

Figure S20 Network of comparisons for vomiting included in the analysis. Cap, capecitabine; CapTra, capecitabine plus trastuzumab; LapCap, lapatinib plus capecitabine; LapVin, lapatinib plus vinorelbine; Ner, neratinib; PerTraCap, pertuzumab plus trastuzumab plus capecitabine; PyrCap, pyrotinib plus capecitabine; TRAE, trastuzumab emtansine; TraEAte, trastuzumab emtansine plus atezolizumab. 


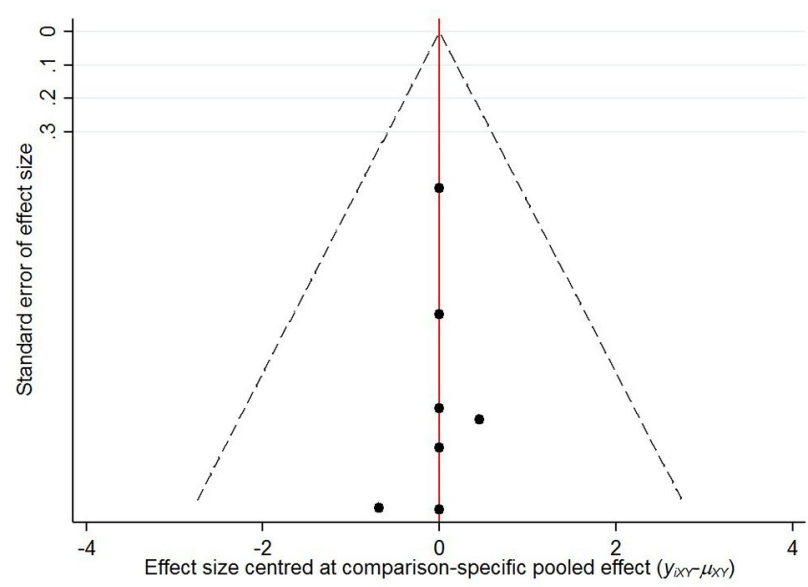

Figure S21 Funnel plot for alanine transaminase increased.

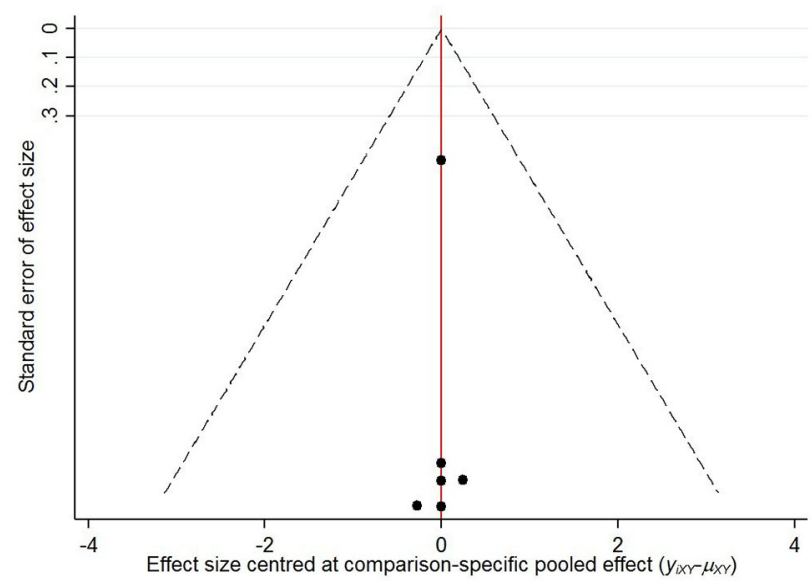

Figure S22 Funnel plot for anemia.

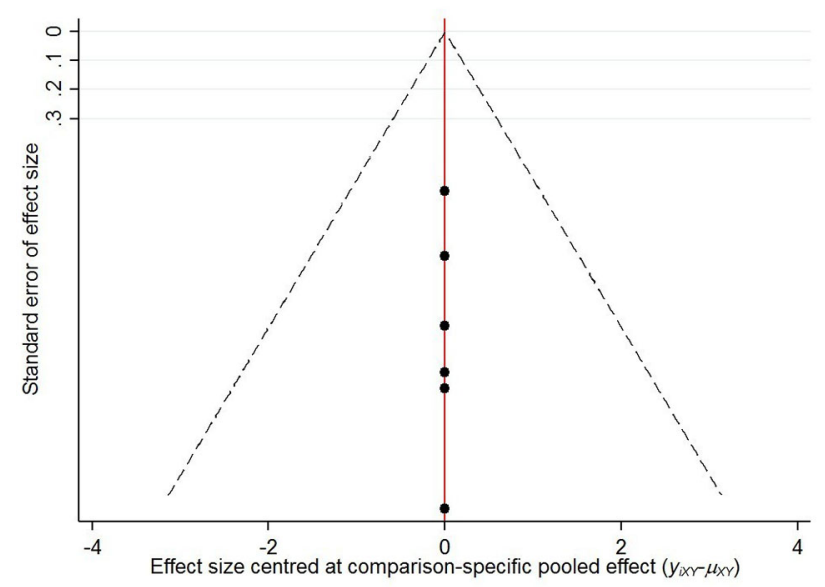

Figure S23 Funnel plot for aspartate aminotransferase increased.

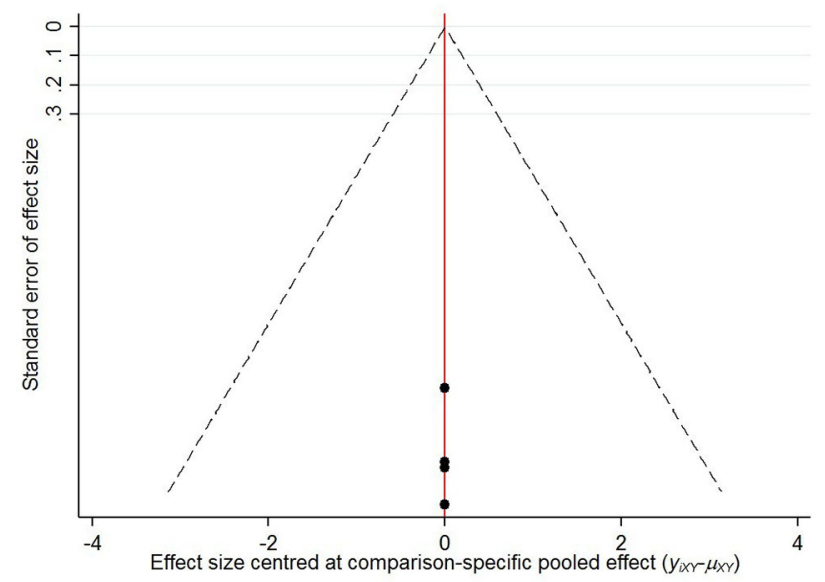

Figure S24 Funnel plot for asthenia. 


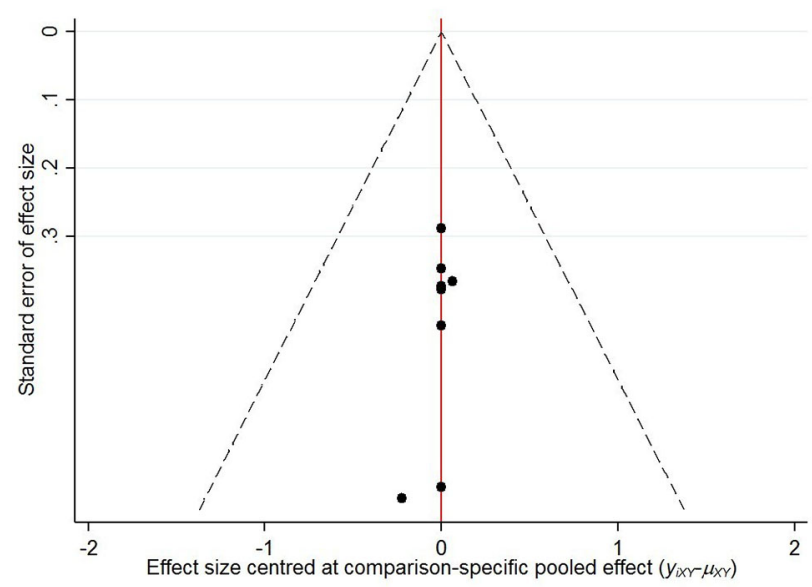

Figure S25 Funnel plot for diarrhea.

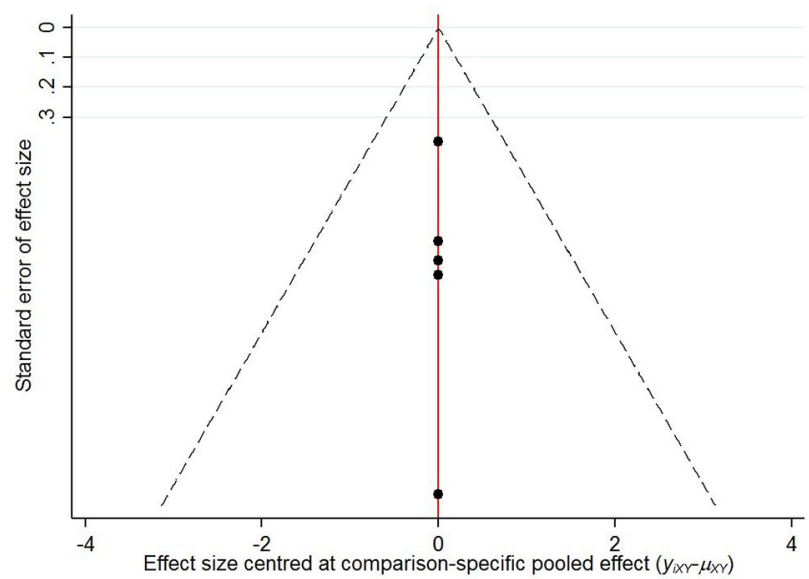

Figure S26 Funnel plot for fatigue.

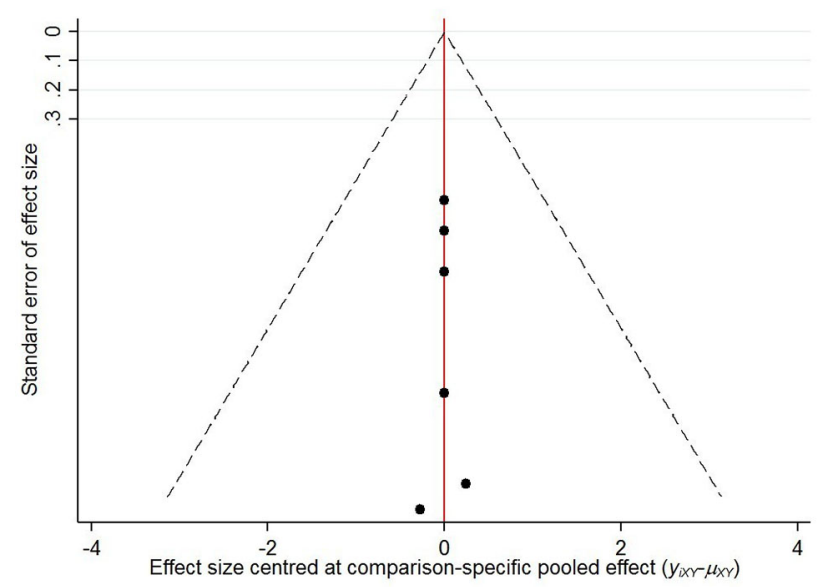

Figure S27 Funnel plot for nausea.

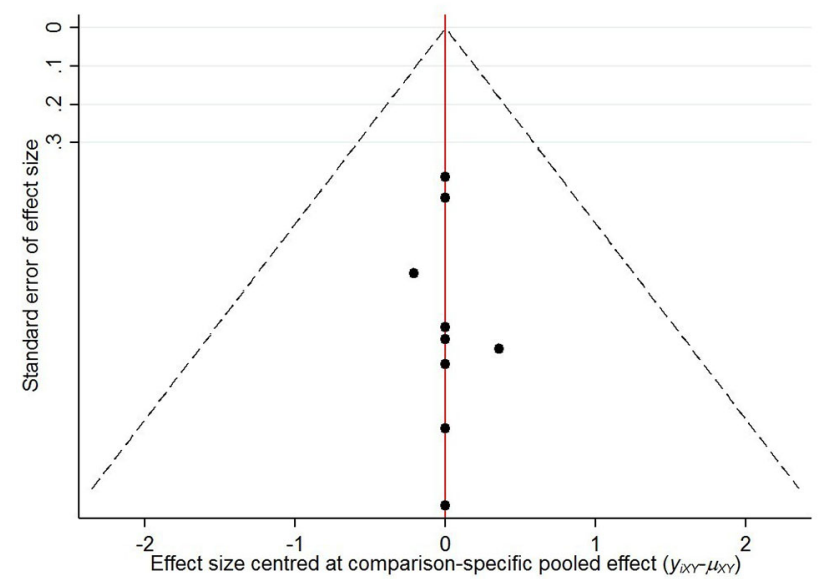

Figure S28 Funnel plot for neutropenia. 


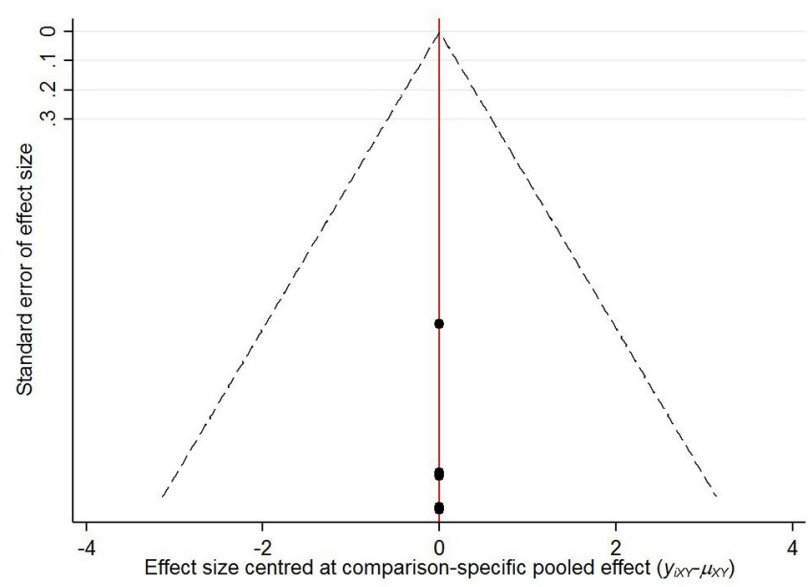

Figure S29 Funnel plot for rash.

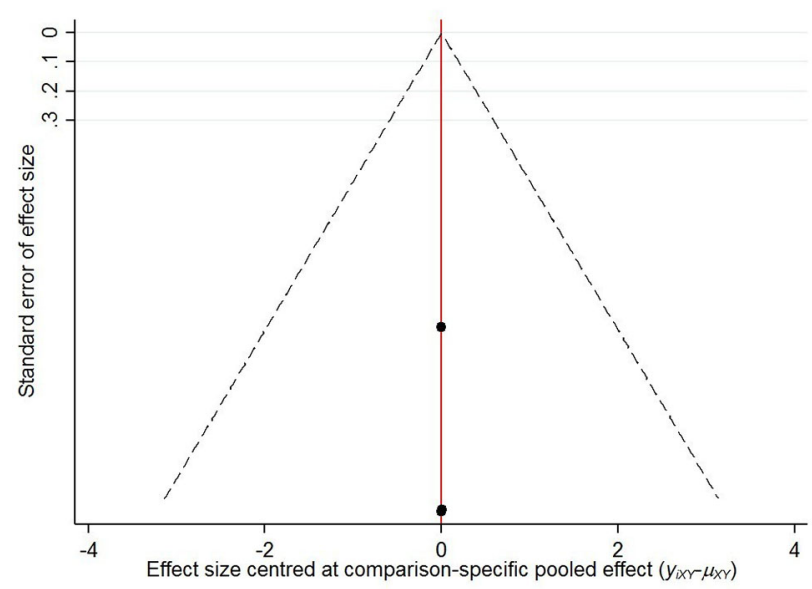

Figure S30 Funnel plot for thrombocytopenia.

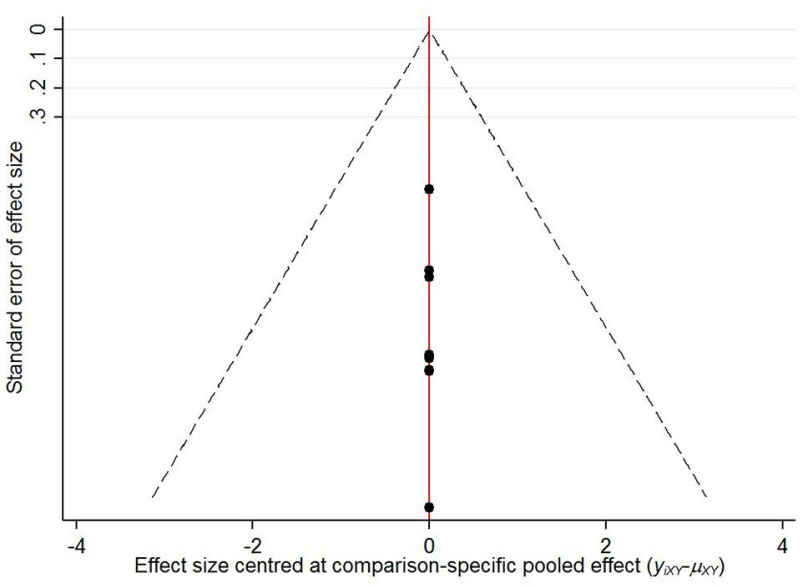

Figure S31 Funnel plot for vomiting. 
Appendix 3 The surface under the cumulative ranking area rank test for grade $\geq 3$ adverse events
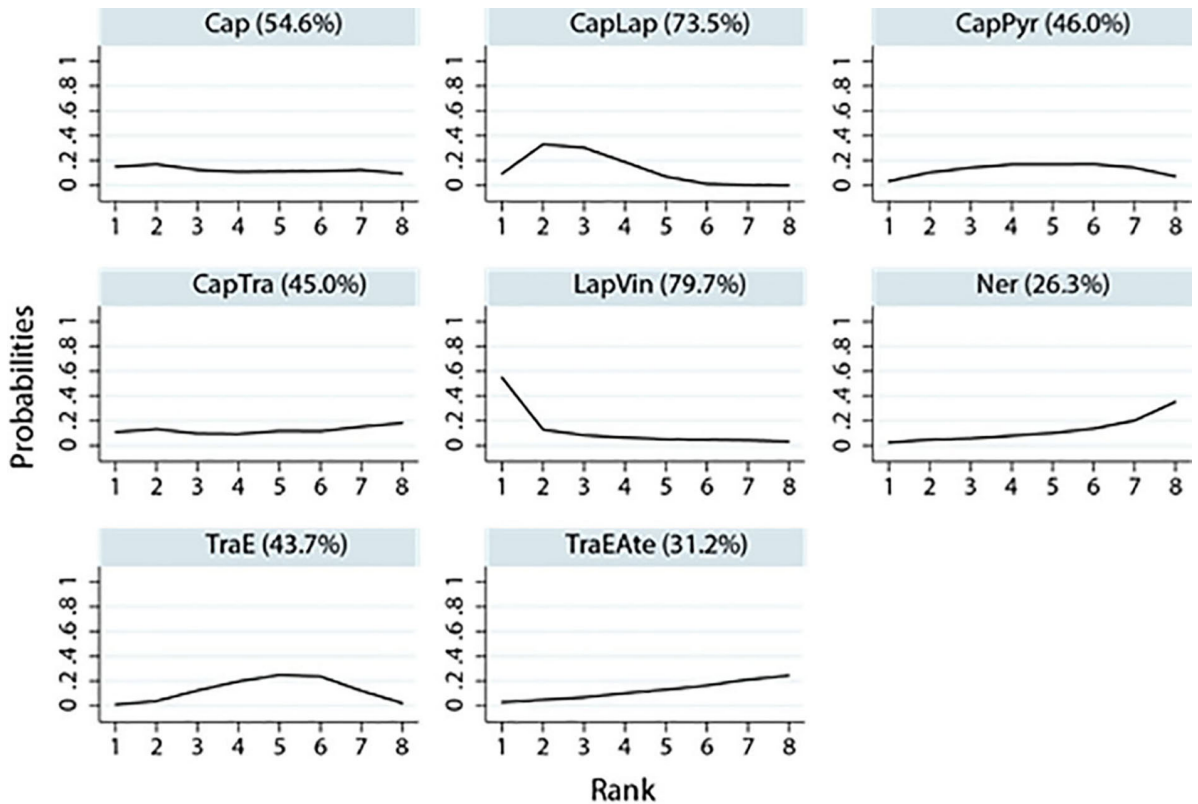

Graphs by Treatment

Figure S1 The surface under the cumulative ranking area rank test for alanine transaminase increased. Cap, capecitabine; Cap Tra, capecitabine plus trastuzumab; LapCa, lapatinib plus capecitabine; LapVin, lapatinib plus vinorelbine; Ner, neratinib; PyrCap, pyrotinib plus capecitabine; TraE, trastuzumab emtansine; TraEAte, trastuzumab emtansine plus atezolizumab.

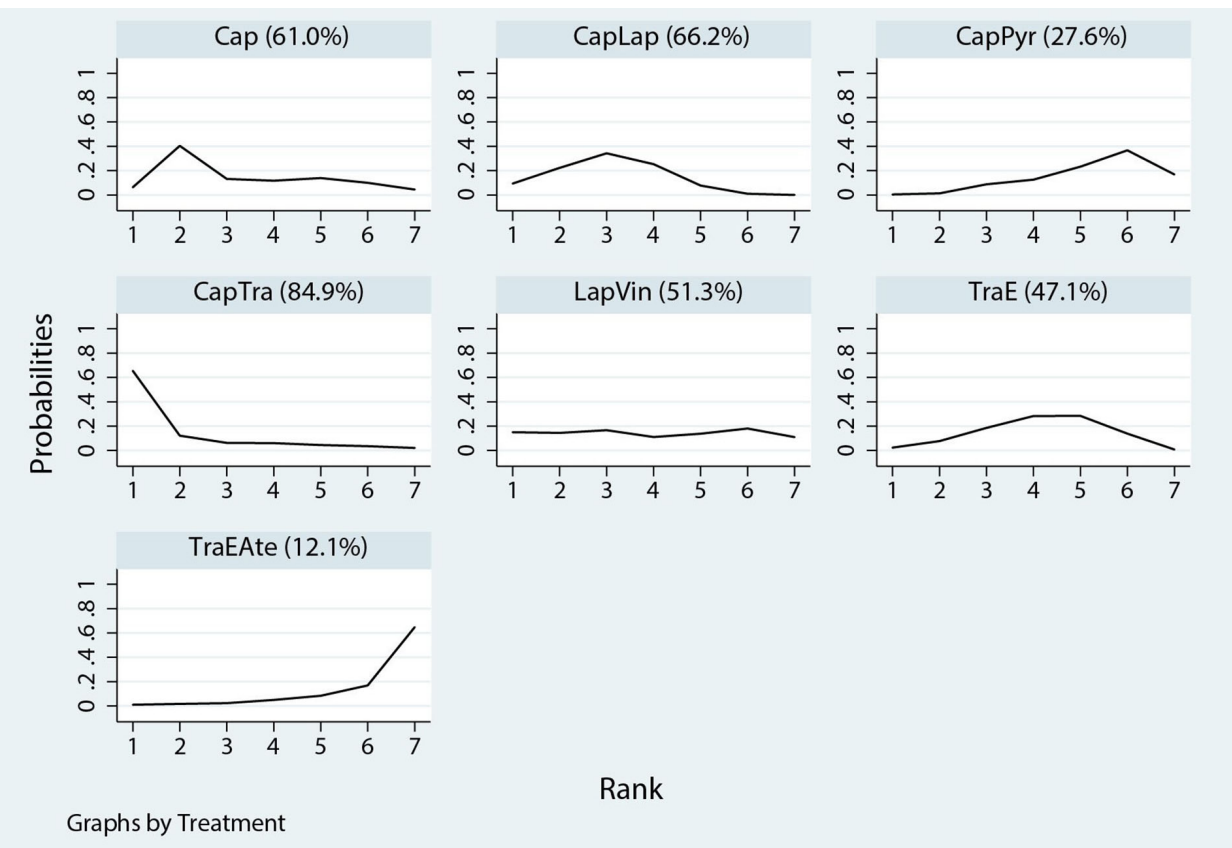

Figure S2 The surface under the cumulative ranking area rank test for anemia. Cap, capecitabine; CapTra, capecitabine plus trastuzumab; LapCap, lapatinib plus capecitabine; LapVin, lapatinib plus vinorelbine; PyrCap, pyrotinib plus capecitabine; TraE, trastuzumab emtansine; TraEAte, trastuzumab emtansine plus atezolizumab. 

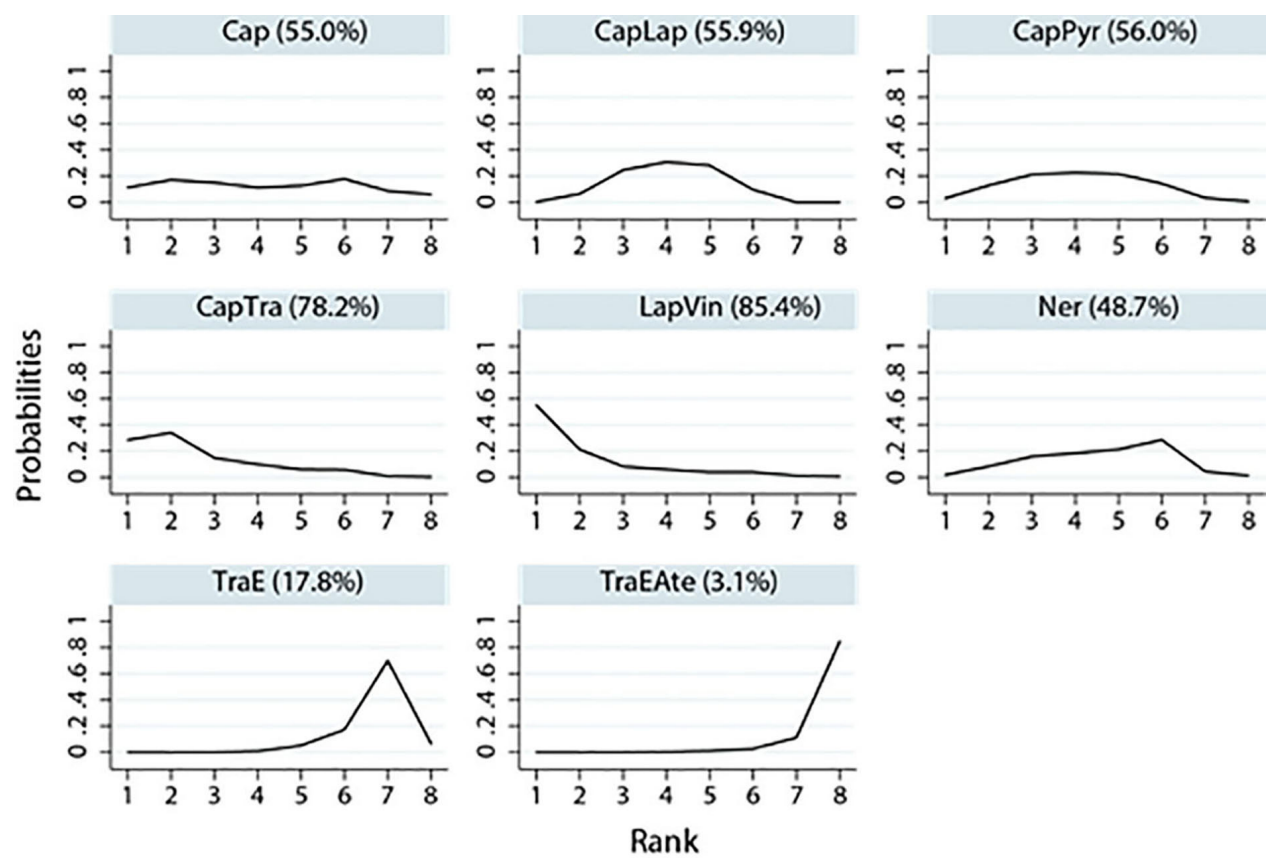

Graphs by Treatment

Figure S3 The surface under the cumulative ranking area rank test for aspartate aminotransferase increased. Cap, capecitabine; CapTra, capecitabine plus trastuzumab; LapCap, lapatinib plus capecitabine; LapVin, lapatinib plus vinorelbine; Ner, neratinib; PyrCap, pyrotinib plus capecitabine; TraE, trastuzumab emtansine; TraEAte, trastuzumab emtansine plus atezolizumab.
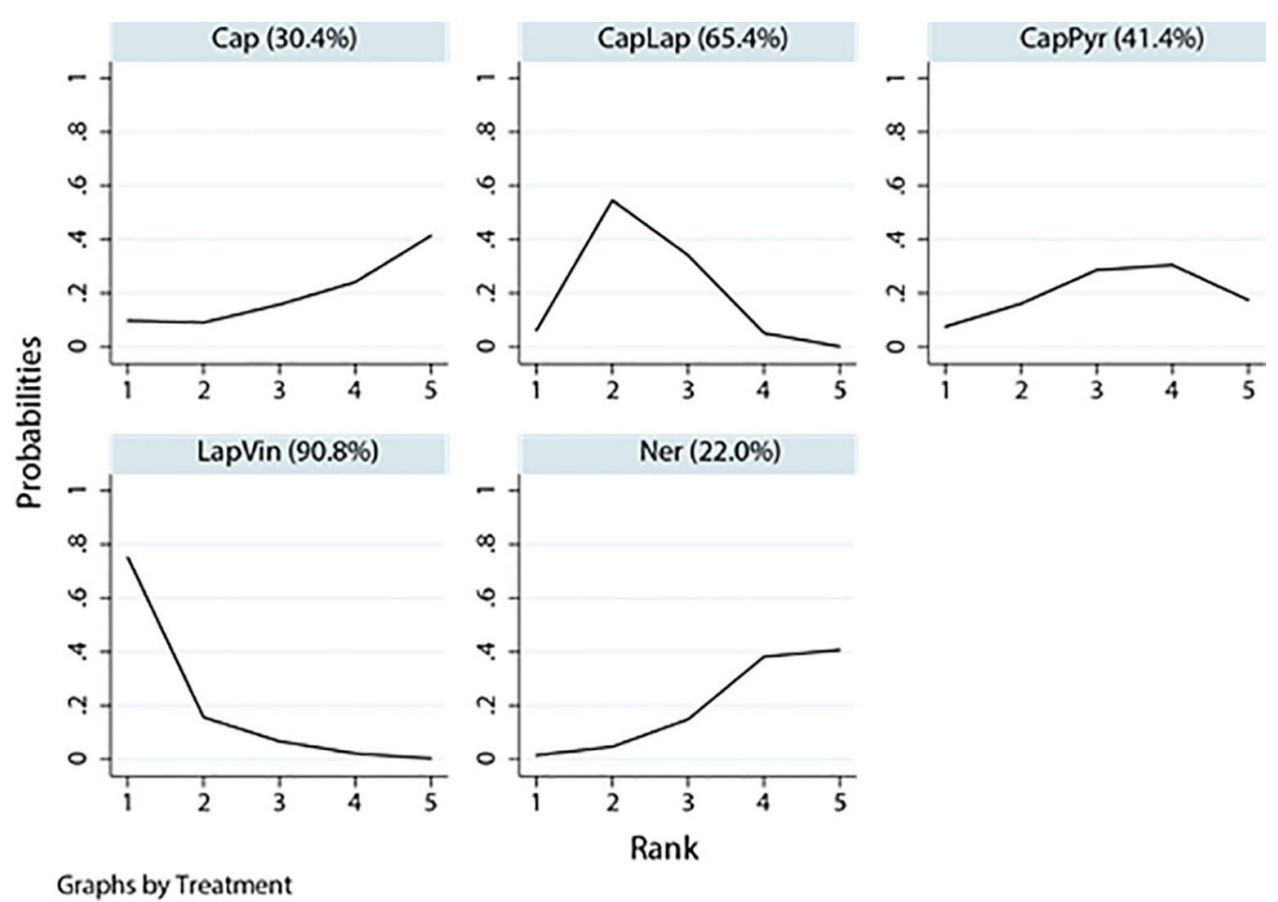

Graphs by Treatment

Figure S4 The surface under the cumulative ranking area rank test for asthenia. Cap, capecitabine; LapCap, lapatinib plus capecitabine; LapVin, lapatinib plus vinorelbine; Ner, neratinib; PyrCap, pyrotinib plus capecitabine. 

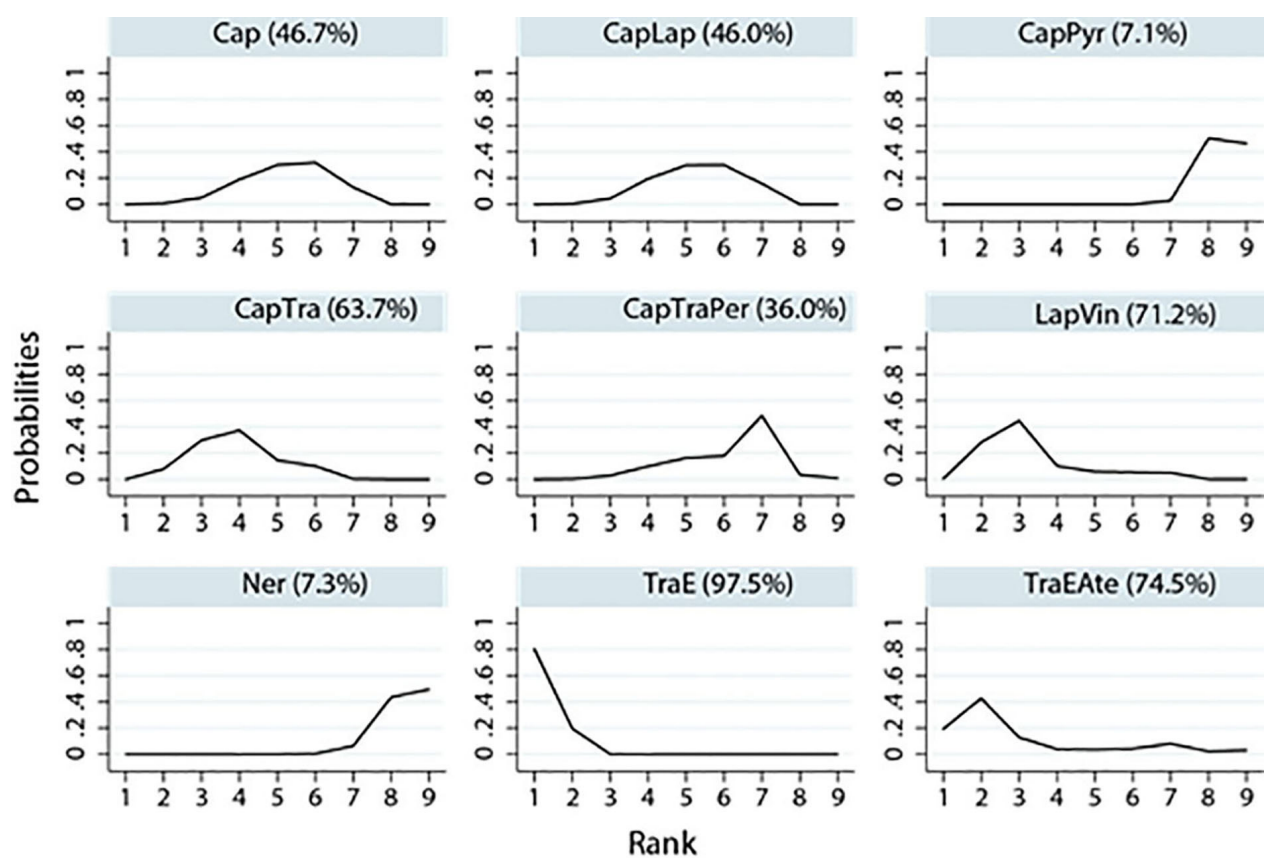

Graphs by Treatment

Figure S5 The surface under the cumulative ranking area rank test for diarrhea. Cap, capecitabine; CapTra, capecitabine plus trastuzumab; LapCap, lapatinib plus capecitabine; LapVin, lapatinib plus vinorelbine; Ner, neratinib; PerTraCap, pertuzumab plus trastuzumab plus capecitabine; PyrCap, pyrotinib plus capecitabine; TraE, trastuzumab emtansine; TraEAte, trastuzumab emtansine plus atezolizumab.
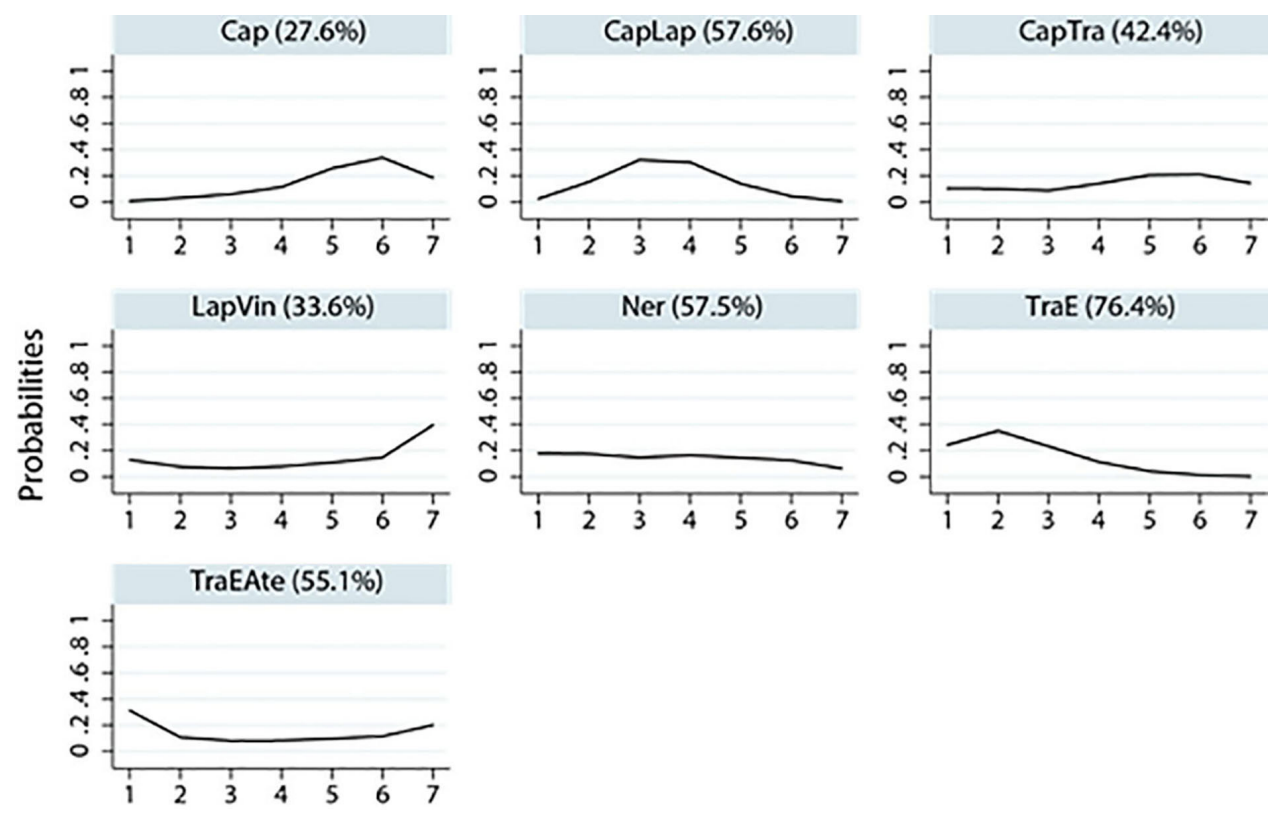

Graphs by Treatment

Rank

Figure S6 The surface under the cumulative ranking area rank test for fatigue. Cap, capecitabine; CapTra, capecitabine plus trastuzumab; LapCap, lapatinib plus capecitabine; LapVin, lapatinib plus vinorelbine; Ner, neratinib; TraE, trastuzumab emtansine; TraEAte, trastuzumab emtansine plus atezolizumab. 

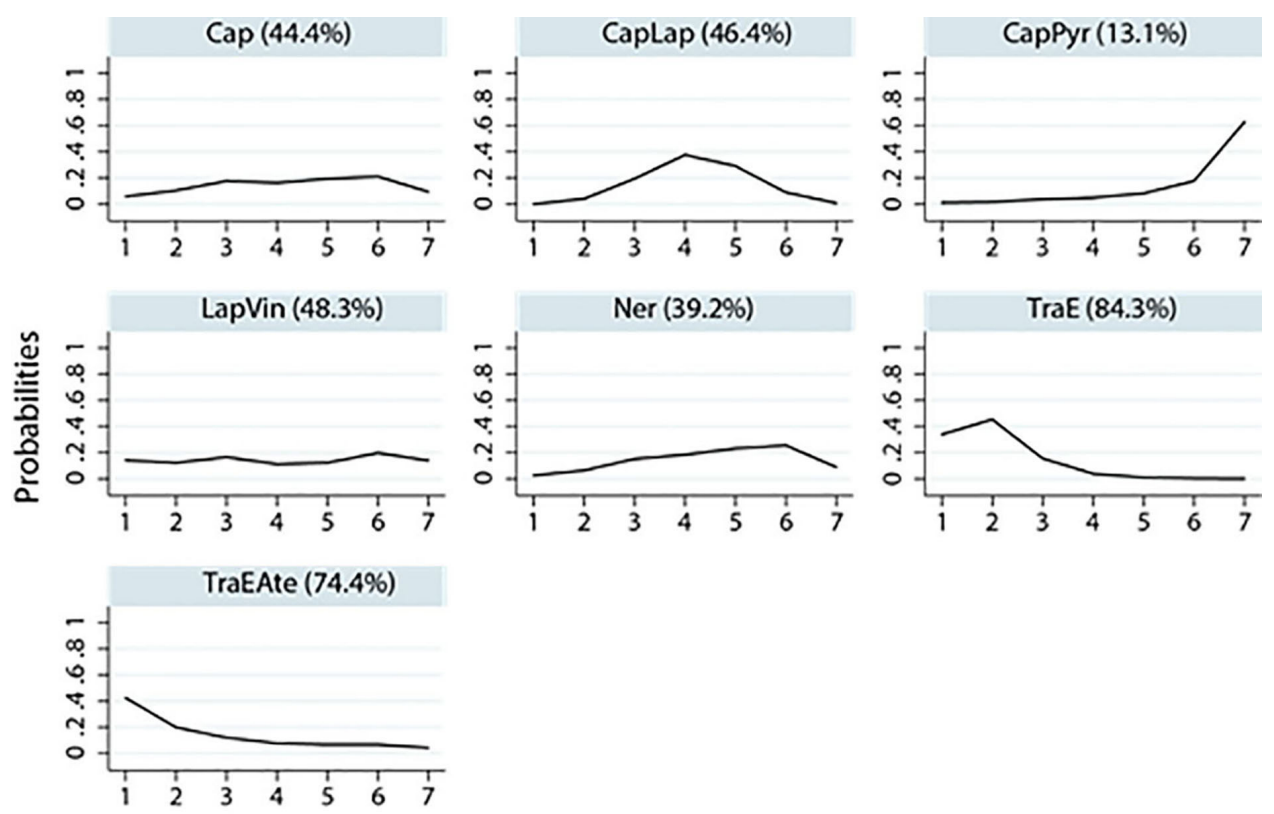

Graphs by Treatment

\section{Rank}

Figure S7 The surface under the cumulative ranking area rank test for nausea. Cap, capecitabine; LapCap, lapatinib plus capecitabine; LapVin, lapatinib plus vinorelbine; Ner, neratinib; PyrCap, pyrotinib plus capecitabine; TraE, trastuzumab emtansine; TraEAte, trastuzumab emtansine plus atezolizumab.
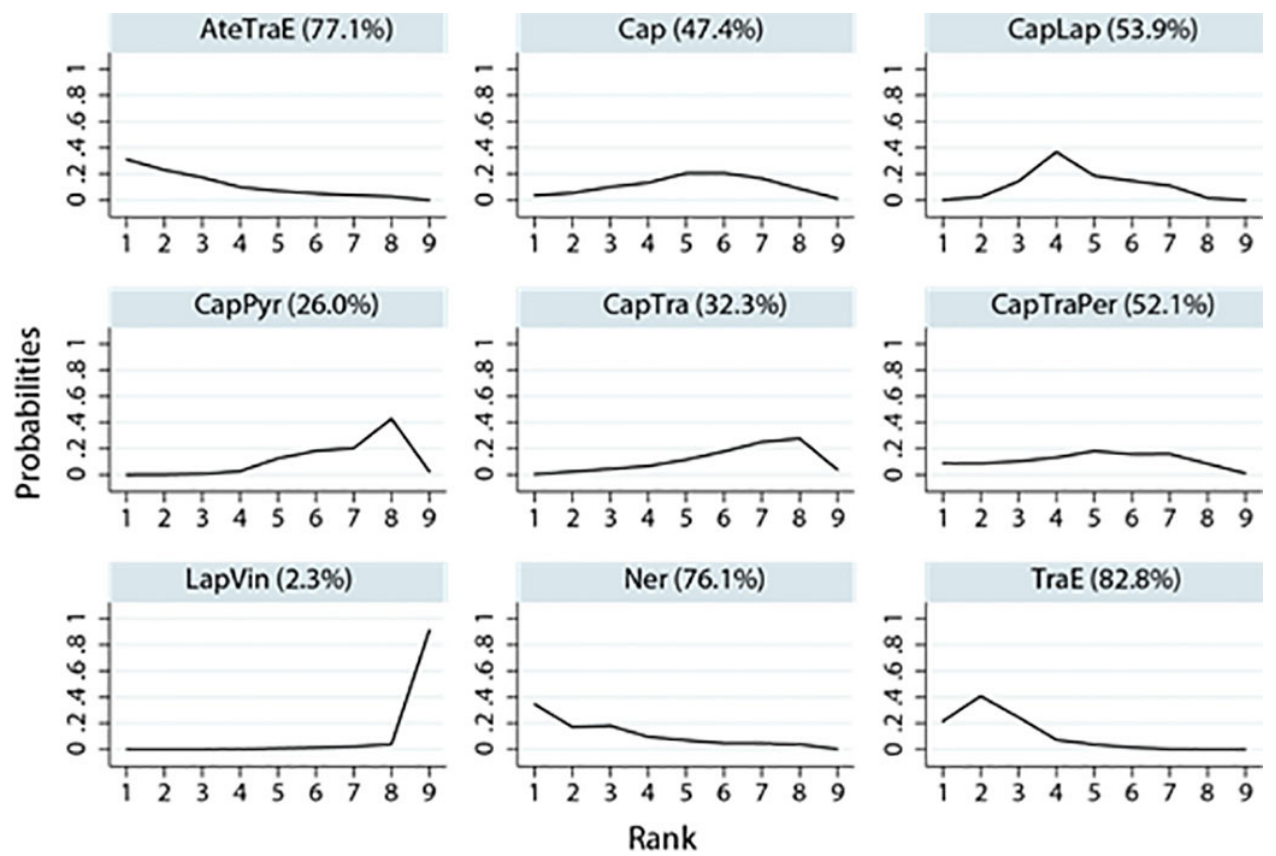

Graphs by Treatment

Figure S8 The surface under the cumulative ranking area rank test for neutropenia. Cap, capecitabine; CapTra, capecitabine plus trastuzumab; LapCap, lapatinib plus capecitabine; LapVin, lapatinib plus vinorelbine; Ner, neratinib; PerTraCap, pertuzumab plus trastuzumab plus capecitabine; PyrCap, pyrotinib plus capecitabine; TraE, trastuzumab emtansine; TraEAte, trastuzumab emtansine plus atezolizumab. 

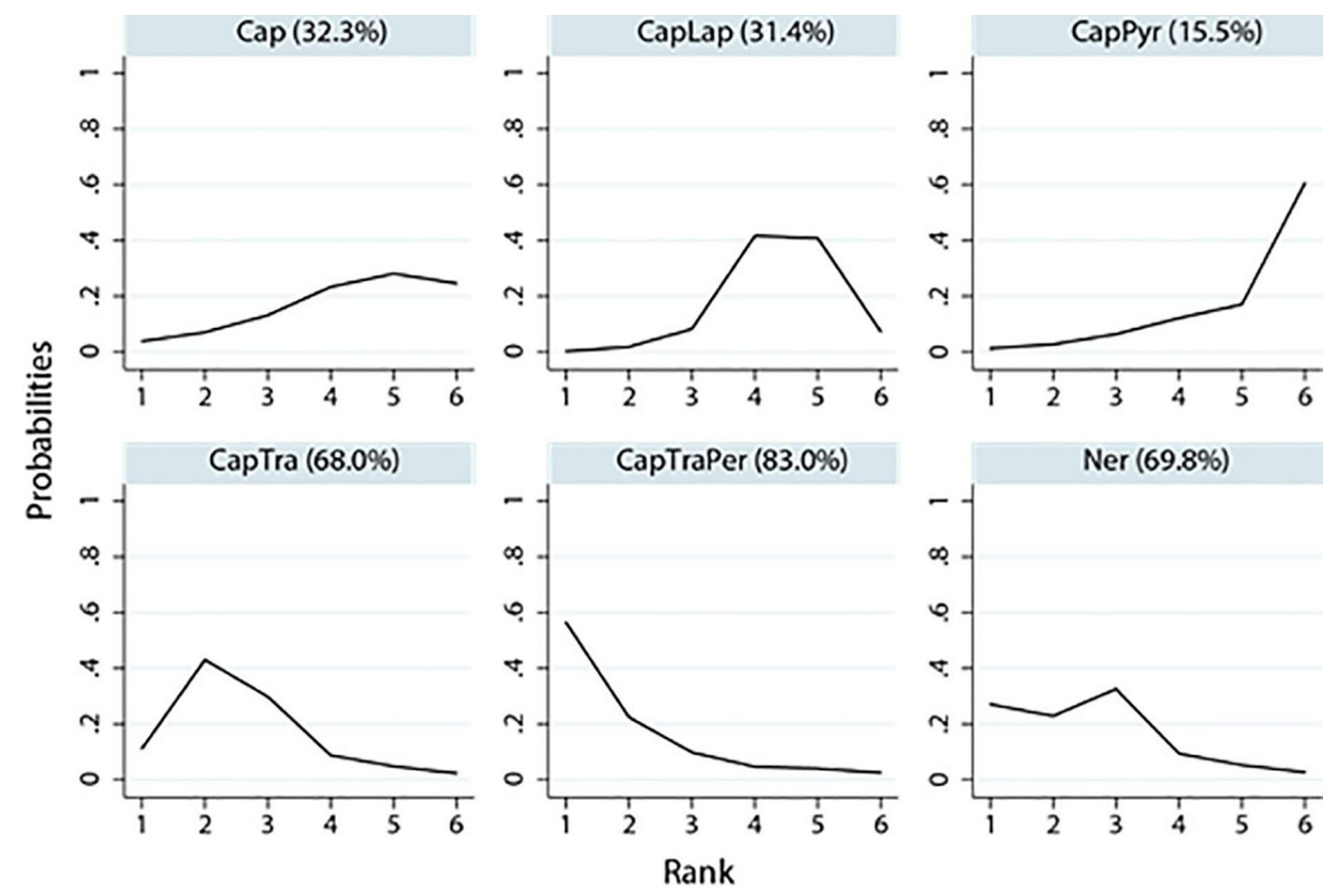

\section{Graphs by Treatment}

Figure S9 The surface under the cumulative ranking area rank test for rash. Cap, capecitabine; CapTra, capecitabine plus trastuzumab; LapCap, lapatinib plus capecitabine; Ner, neratinib; PerTraCap, pertuzumab plus trastuzumab plus capecitabine; PyrCap, pyrotinib plus capecitabine.
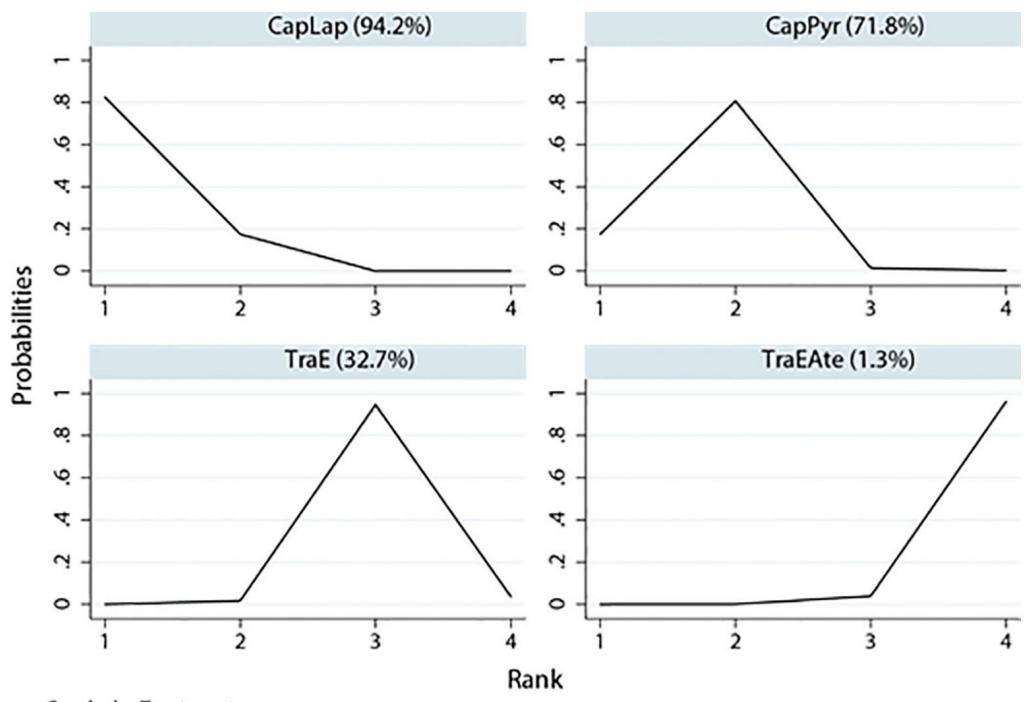

Graphs by Treatment

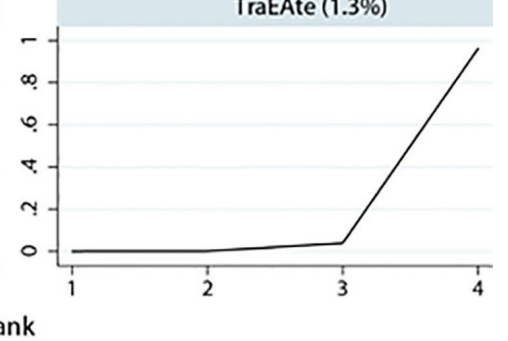

Figure S10 The surface under the cumulative ranking area rank test for thrombocytopenia. LapCap, lapatinib plus capecitabine; PyrCap, pyrotinib plus capecitabine; TraE, trastuzumab emtansine; TraEAte, trastuzumab emtansine plus atezolizumab. 

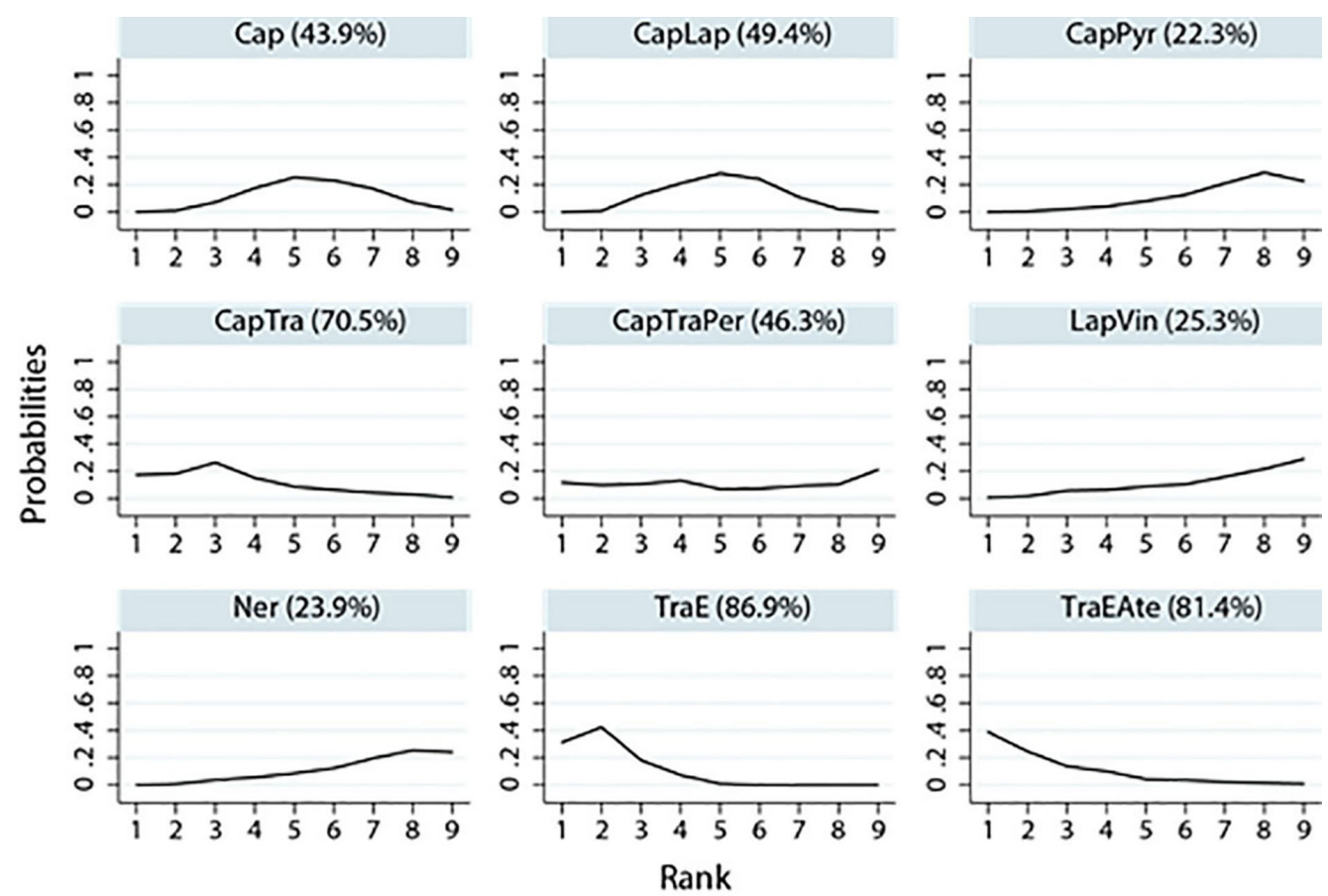

Graphs by Treatment

Figure S11 The surface under the cumulative ranking area rank test for vomiting. Cap, capecitabine; CapTra, capecitabine plus trastuzumab; LapCap, lapatinib plus capecitabine; LapVin, lapatinib plus vinorelbine; Ner, neratinib; PerTraCap, pertuzumab plus trastuzumab plus capecitabine; PyrCap, pyrotinib plus capecitabine; TraE, trastuzumab emtansine; TraEAte, trastuzumab emtansine plus atezolizumab. 